\title{
Raman Scattering in a Two-Layer Antiferromagnet
}

\author{
Dirk K. Morr, ${ }^{1}$ Andrey V. Chubukov, ${ }^{1,2}$ Arno P. Kampf, ${ }^{3}$ and G. Blumberg ${ }^{4,5}$ \\ 1 Department of Physics, University of Wisconsin-Madison, \\ 1150 University Avenue, Madison, Wisconsin 53706 \\ ${ }^{2}$ P.L. Kapitza Institute for Physical Problems, Moscow, Russia \\ ${ }^{3}$ Institut für Theoretische Physik, Universität zu Köln, Zülpicher Str. 77, \\ 50937 Köln, Germany \\ ${ }^{4}$ NSF Science and Technology Center for Superconductivity and \\ Department of Physics, University of Illinois at Urbana-Champaign, \\ 1110 W. Green Str. Urbana IL 61801 \\ ${ }^{5}$ Institute of Chemical Physics and Biophysics, Rävala 10, Tallinn EE0001, Estonia
} (September 19, 2018)

\begin{abstract}
Two-magnon Raman scattering is a useful tool to verify recent suggestions concerning the value of the interplanar exchange constant in antiferromagnetic two-layer systems, such as $\mathrm{YBa}_{2} \mathrm{Cu}_{3} \mathrm{O}_{6+x}$. We present a theory for Raman scattering in a two-layer antiferromagnet. We study the spectra for the electronic and magnetic excitations across the charge transfer gap within the one-band Hubbard model and derive the matrix elements for the Raman scattering cross section in a diagrammatic formalism. We analyze the effect of the interlayer exchange coupling $J_{2}$ for the Raman spectra in $A_{1 g}$ and $B_{1 g}$ scattering geometries both in the non-resonant regime (when the Loudon-Fleury model is valid), and at resonance. We show that within the Loudon-Fleury approximation, a nonzero $J_{2}$ gives rise to a finite signal in $A_{1 g}$ scattering geometry. Both, in this approximation and at resonance, the
\end{abstract}


intensity in the $A_{1 g}$ channel has a peak at small transferred frequency equal to twice the gap in the spin-wave spectrum. We compare our results with experiments in $\mathrm{YBa}_{2} \mathrm{Cu}_{3} \mathrm{O}_{6.1}$ and $\mathrm{Sr}_{2} \mathrm{CuO}_{2} \mathrm{Cl}_{2}$ compounds and argue that the large value of $J_{2}$ suggested in a number of recent studies is incompatible with Raman experiments in $A_{1 g}$ geometry.

Typeset using REVTEX 


\section{INTRODUCTION}

Since the discovery of high- $T_{c}$ superconductivity [1] a lot of work has been done in an attempt to understand the pairing mechanism. Most of the existing theories consider boson-mediated pairing between electrons in the same $\mathrm{CuO}_{2}$ plane, but there also exist arguments in favor of the pairing between electrons in adjacent $\mathrm{CuO}_{2}$ planes [2]. These arguments are mostly applied to $\mathrm{YBa}_{2} \mathrm{Cu}_{3} \mathrm{O}_{6+x}(\mathrm{YBCO})$ compounds in which the unit cell contains pairs of $\mathrm{CuO}_{2}$ planes separated by a charge reservoir. A strong magnetic coupling between the planes of a bilayer was also suggested [3] [5] as a possible source for an experimentally observed strong downturn renormalization of the low temperature Pauli susceptibility of holes in underdoped $Y B C O$ compounds ("spin-gap" phenomenon) as well as for the maximum in the spin-lattice relaxation rate at temperatures well above $T_{c}$ [6].

An essential input parameter for these theories is the value of the interplane hopping amplitude or the $\mathrm{Cu}-\mathrm{Cu}$ superexchange interaction between the two $\mathrm{CuO}_{2}$ planes. In this paper, we will argue that the Raman scattering experiments in two-layer compounds allow an estimate for the value of the interlayer exchange coupling.

The Raman scattering in single-layer parent high- $T_{c}$ compounds has been intensively studied over the last few years [7] [12]. A large number of studies has been performed to understand the Raman spectra in different scattering geometries. In $B_{1 g}$ geometry $\left(\mathbf{e}_{i}=\right.$ $(\hat{x}+\hat{y}) / \sqrt{2}, \mathbf{e}_{f}=(\hat{x}-\hat{y}) / \sqrt{2}$ [13], where $\mathbf{e}_{i, f}$ are polarization unit vectors of the incident and scattered photons) the dominant feature of the magnetic Raman intensity profile is a peak at about $3000 \mathrm{~cm}^{-1}$, which is attributed to a two-magnon scattering process [14. The two-magnon peak has been observed in all parent high- $T_{c}$ compounds. Besides, the

experiments also found a strong Raman signal in the $A_{1 g}$ geometry $\left(\mathbf{e}_{i}=\mathbf{e}_{f}=(\hat{x}+\hat{y}) / \sqrt{2}\right)$ [15]. The $A_{1 g}$ signal has a maximum at about the same frequency as in $B_{1 g}$ geometry, but the width of the peak is larger and its intensity is about a quarter as strong.

A traditional framework for the understanding of Raman experiments is the LoudonFleury theory [16] which describes the interaction of light with only spin degrees of freedom. 
This theory explains a peak in $B_{1 g}$ geometry but predicts that there should be no scattering in $A_{1 g}$ geometry. Recently, however, it was found [12] that the Loudon-Fleury approach has to be modified because Raman experiments are mostly performed near the resonant regime where photon frequencies are close to the charge transfer gap of the insulating compounds, and one can by no means neglect electronic degrees of freedom. In this regime, the diagrams which are neglected in the Loudon-Fleury theory, and which contribute to both $A_{1 g}$ and $B_{1 g}$ scattering are actually more important than the diagrams included in the Loudon-Fleury theory.

In this paper, we will study magnetic Raman scattering in two-layer antiferromagnetic insulators. We will show that in the presence of the interlayer exchange coupling, $J_{2}$, the Raman scattering profile in the $A_{1 g}$ scattering geometry acquires qualitatively new features. In particular, a nonzero $J_{2}$ gives rise to a nonzero Raman intensity in the $A_{1 g}$ scattering geometry already in the Loudon-Fleury approximation. Moreover, we will see that within the Loudon-Fleury theory, there is a very strong enhancement of $A_{1 g}$ intensity at small transferred frequencies $\omega_{i}-\omega_{f}=\delta \omega^{\text {res }} \approx 4\left(J_{1} J_{2}\right)^{1 / 2}$, where $\omega_{i}$ and $\omega_{f}$ are the frequencies of the incoming and the scattered photon, respectively, and $J_{1}$ is the intralayer exchange coupling. At the frequency shift $\delta \omega^{\text {res }}$, the intensity in the $A_{1 g}$ channel actually turns out to be larger than the intensity in the $B_{1 g}$ channel, which, as we recall, is nonzero already in the absence of $J_{2}$. In the resonant regime, when the incident photon frequency becomes comparable to the single particle excitation gap of the insulator, there exists substantial $A_{1 g}$ scattering already for a single layer. Nevertheless, we will argue that even in this situation, in a two-layer system, there is a measurable change of the Raman intensity near the frequency shift $\delta \omega^{\text {res }}$. We will also consider the scattering in $B_{1 g}$ geometry and will show that in this geometry the effect of $J_{2}$ is much weaker than for $A_{1 g}$ scattering.

In principle, the presence of the new features in the $A_{1 g}$ Raman scattering allows one to find the value of the interlayer coupling. In reality, however, in $Y B C O$, the frequency shift $\delta \omega^{r e s}$ is in the region where the dominant contribution to the Raman intensity comes from phonon rather than two-magnon scattering. However, we will argue that there are 
still several features of the Raman profile in $Y B C O$ which are absent in the single-layer compound $\mathrm{Sr}_{2} \mathrm{CuO}_{2} \mathrm{Cl}_{2}$ and which allow to find an estimate for $J_{2}$. We found from our analysis that $J_{2}$ is likely to be about $0.1 J_{1}$. More rigorously, we can place the upper boundary for $J_{2}$ as $J_{2} \sim 0.25 \mathrm{~J}$. Neutron scattering data for the spin wave velocity $c_{s w}$ at half-filling [17] yield $J_{1} \sim 120 \mathrm{meV}$. As $c_{s w}$ is only weakly dependent on $J_{2}$ for small $J_{2} / J_{1}$, this implies that the probable value is $J_{2} \sim 12 \mathrm{meV}$, and the upper boundary for $J_{2}$ is $30 \mathrm{meV}$. This is consistent with the estimate $5 \mathrm{meV}<J_{2}<20 \mathrm{meV}$ for $J_{2}$ extracted from the analysis of NMR data [18] on the double-layer material $\mathrm{Y}_{2} \mathrm{Ba}_{4} \mathrm{Cu}_{7} \mathrm{O}_{15}$ [19], but substantially smaller than $J_{2} \sim 0.55 J_{1}$ inferred from infrared transmission and reflection measurements [20] and also substantially smaller than the theoretical estimate $J_{2}=56 \mathrm{meV}$ by Barriquand and Sawatzky [21].

The paper is organized as follows. We start in Sec. II with the Hubbard model at half-filling which has long range antiferromagnetic order in its ground state, and derive in

a diagrammatic formalism the effective Loudon-Fleury model for Raman scattering in $A_{1 g}$ and $B_{1 g}$ geometries in the non-resonant regime (i.e. assuming that the photon frequencies are smaller than the Mott-Hubbard gap). In Sec. [II, we use this model to compute the Raman intensity in the $A_{1 g}$ and $B_{1 g}$ channels first without magnon-magnon interaction, and then by including multiple scattering between magnons. In Sec. IV, we will discuss the resonant regime. Finally, in Sec. $\mathrm{V}$, we summarize our results and discuss them in the context of experimental data for single-layer $\mathrm{Sr}_{2} \mathrm{CuO}_{2} \mathrm{Cl}_{2}$ and double-layer $\mathrm{YBa}_{2} \mathrm{Cu}_{3} \mathrm{O}_{6.1}$.

\section{THE DERIVATION OF THE LOUDON-FLEURY HAMILTONIAN FOR THE NON-RESONANT CASE}

In this section we derive the effective Loudon-Fleury model for Raman scattering using a momentum space diagrammatic formalism. This formalism has recently been applied to derive the Loudon-Fleury Hamiltonian for a single-layer system [12]. Technically, the calculations for two-layer systems are more involved as one has to double the number of 
fermionic operators. Conceptually, however, our approach is exactly the same as in Ref. [12, and we therefore refrain from discussing the calculational steps in full length.

The starting point of our calculations is the simplest one-band Hubbard Hamiltonian for a two-layer system at half-filling on a square lattice given by:

$$
H=-t \sum_{<i, j>}\left(c_{i, \sigma}^{\dagger} c_{j, \sigma}+d_{i, \sigma}^{\dagger} d_{j, \sigma}+\text { h.c. }\right)-t^{\prime} \sum_{i}\left(c_{i, \sigma}^{\dagger} d_{i, \sigma}+\text { h.c. }\right)+U \sum_{i, \alpha} n_{i, \alpha, \uparrow} n_{i, \alpha, \downarrow}
$$

where the $c$ and $d$ operators represent the electrons of layer 1 and 2, respectively, $\alpha=1,2$, $n_{i, 1, \sigma}=c_{i, \sigma}^{\dagger} c_{i \sigma}, n_{i, 2, \sigma}=d_{i, \sigma}^{\dagger} d_{i, \sigma}$, and $t^{\prime}$ is the hopping amplitude between the planes for which we assume $t^{\prime}<t$ (see Fig. [1). We will also assume that $t / U \ll 1$ and thus perform our calculations only to leading order in $t / U$. This large $U$ one-band model with only nearestneighbor in-plane hopping is indeed a simplification, but it was argued in [12] that this model already contains the relevant physics for the analysis of Raman scattering in $Y B C O$ compounds. Here we follow this reasoning and assume that the model of Eq. (1) is valid.

The mechanism of two-magnon Raman scattering is straightforward and has been discussed a number of times in the literature [22]: the incoming photon with frequency $\omega_{i}$ creates a virtual particle-hole pair, which then in turn emits two magnons with momenta $\mathbf{q}$ and $-\mathbf{q}$ before it annihilates into an outgoing photon with frequency $\omega_{f}$. For the diagrammatic calculation of the required matrix element for Raman scattering we thus need to compute two types of vertices: the vertices for the interaction between the electrons and the electromagnetic vector potential of the photons and the vertices for the interaction between the electrons and the magnons.

The procedure to derive the coupling of light to the electrons was previously described by Shastry and Shraiman [23]: the photons introduce a slowly varying vector potential $\mathbf{A}(\mathbf{r}, t)$ in the presence of which the hopping term in the kinetic energy of the electrons acquires a phase $\left(i \frac{e}{\overline{\hbar c}} \int_{i}^{j} \mathbf{A}(\mathbf{l}, t) \cdot d \mathbf{l}\right)$. The Hubbard Hamiltonian is then expanded to leading orders in $\mathbf{A}$. One further introduces the staggered magnetization as the spin density wave (SDW) order parameter and transforms to new fermionic operators which diagonalize the Hartree-Fock factorized Hamiltonian [24]. To derive the interaction vertex between fermions and magnons, 
one has to compute the transverse spin susceptibilities with and without momentum transfer $\mathbf{Q}=(\pi, \pi)$, and construct the interaction Hamiltonian which reproduces all dynamic spin susceptibilities. This procedure is unique though rather involved for a two-layer system. We will skip the details and present only our final results (some of the useful formulae are collected in Appendix A).

In terms of the new SDW quasiparticle operators, the Hubbard Hamiltonian takes the form

$$
H=\sum_{\mathbf{k}, \sigma}^{\prime}\left\{E_{\mathbf{k}}^{+}\left(a_{\mathbf{k}, \sigma}^{\dagger} a_{\mathbf{k}, \sigma}-b_{\mathbf{k}, \sigma}^{\dagger} b_{\mathbf{k}, \sigma}\right)+E_{\mathbf{k}}^{-}\left(e_{\mathbf{k}, \sigma}^{\dagger} e_{\mathbf{k}, \sigma}-f_{\mathbf{k}, \sigma}^{\dagger} f_{\mathbf{k}, \sigma}\right)\right\}
$$

where

$$
E_{\mathbf{k}}^{ \pm}=\sqrt{\left(\epsilon_{\mathbf{k}}^{ \pm}\right)^{2}+\Delta^{2}}, \quad \epsilon_{\mathbf{k}}^{ \pm}=-4 t \nu_{\mathbf{k}} \pm t^{\prime}, \quad \nu_{\mathbf{k}}=\frac{\cos k_{x}+\cos k_{y}}{2}
$$

and we set the lattice constant $a_{0}=1$. Here and below, the prime in the summation sign indicates that the summation is restricted to the magnetic Brillouin zone, i.e. to momenta where $\nu_{\mathbf{k}}>0$. For a two-layer system we obtain two pairs of conduction (described by the $a$ and $e$ ) and valence ( $b$ and $f$ ) operators. The energy dispersions for each pair are shifted by $\mathbf{Q}=(\pi, \pi)$. The direct gap $2 \Delta$ between the bands is determined by a self-consistency equation for the staggered magnetization and reduces to $2 \Delta=U$ in the strong coupling limit.

The interaction between the fermionic current and the vector potential of light, which is relevant for two-magnon scattering at transferred photon frequencies small compared to the Mott-Hubbard gap, has the form

$$
H_{j}=-\frac{e}{\hbar c} \sum_{\mathbf{q}} \mathbf{j}_{\mathbf{q}} \cdot \mathbf{A}_{-\mathbf{q}}
$$

$\mathbf{j}_{\mathbf{q}}$ is the current operator whose momentum can be safely set to zero since the velocity of light is several orders of magnitude larger than the Fermi velocity. To lowest order in $t / U$ the components of the current operator are given by

$$
j_{\mathbf{q}=0}^{\alpha}=\sum_{\mathbf{k}, \sigma}^{\prime} \frac{\partial \epsilon_{\mathbf{k}}}{\partial k_{\alpha}}\left[a_{\mathbf{k}, \sigma}^{\dagger} b_{\mathbf{k}, \sigma}+e_{\mathbf{k}, \sigma}^{\dagger} f_{\mathbf{k}, \sigma}+h . c .\right] \text {. }
$$


We see that to lowest order in $t / U$ the interaction with light only leads to excitations of quasiparticles between the valence and conduction bands of each pair. Excitations within each band are of higher order in $t / U$ and are therefore neglected in our present strong coupling theory. It is noteworthy that the fermionic current rewritten in terms of quasiparticles which decouple the Hamiltonian into two separate terms (i.e., $\alpha$ and $\beta$ operators introduced in (A3)), contains only densities of these fermionic quasiparticles. As a result, there are no terms in $j_{\mathbf{q}=0}$ which would correspond to excitations between the valence band of one pair and the conduction band of the other pair (e.g. $a_{\mathbf{k}, \sigma}^{\dagger} f_{\mathbf{k}, \sigma}$ ) 225].

We now present the result for the Hamiltonian which describes the magnon-fermion interaction. A systematic way to derive this Hamiltonian is to extend the Hubbard model to a large number of $n=2 S$ orbitals at a given site, and use a $1 / S$ expansion [26,27]. The resulting spin-wave spectrum is that for a spin $S$ antiferromagnet. In this Section, we will consider only noninteracting spin waves, i.e. we will keep only the leading linear term in the $1 / S$ expansion. To simplify notations we will, however, not keep the overall factors of $S$ in the formulae and thus present the results for $n=2 S=1$.

The spin-wave excitation spectrum of the two-layer antiferromagnet consists of one doubly degenerate branch with the dispersion [28]

$$
\Omega_{1}(\mathbf{q})=2 J_{1} \sqrt{\left(1-\nu_{\mathbf{q}}\right)\left(1+\nu_{\mathbf{q}}+J_{2} / 2 J_{1}\right)}
$$

for momenta $\mathbf{q}$ in the first Brillouin zone. The dynamic transverse spin susceptibility has poles at $\Omega=\Omega_{1}(\mathbf{q})$ and $\Omega=\Omega_{1}(\mathbf{q}+\mathbf{Q})$. For a single-layer antiferromagnet, $\Omega_{1}(\mathbf{q})=$ $\Omega_{1}(\mathbf{q}+\mathbf{Q})$, and the two poles are indistinguishable. For two-layer systems, however, $\Omega_{1}(\mathbf{q})$ and $\Omega_{1}(\mathbf{q}+\mathbf{Q})$ are different, and it is convenient to introduce two types of magnon operators $m_{\mathbf{q}}$ and $n_{\mathbf{q}}$ with the dispersions $\Omega_{1}(\mathbf{q})$ and $\Omega_{2}(\mathbf{q})=\Omega_{1}(\mathbf{q}+\mathbf{Q})$.

The electron-magnon interaction Hamiltonian can be obtained in the same way as for a single-layer antiferromagnet [12]: it is uniquely defined by the requirement that it should reproduce the forms of the dynamic spin susceptibilities, both with zero momentum transfer and with momentum transfer $\mathbf{Q}$. The susceptibilities are presented in Appendix B, and the 
interaction Hamiltonian which reproduces them has the form

$$
\begin{aligned}
& H_{e l-m a g}=\sum_{\mathbf{k}}^{\prime} \sum_{\mathbf{q}, \sigma}\left[a_{\mathbf{k}+\mathbf{q}, \sigma}^{\dagger} a_{\mathbf{k},-\sigma} m_{-\mathbf{q}}^{\dagger} \Gamma_{-+}(\mathbf{k}, \mathbf{q})+e_{\mathbf{k}+\mathbf{q}, \sigma}^{\dagger} e_{\mathbf{k},-\sigma} m_{-\mathbf{q}}^{\dagger} \Gamma_{--}(\mathbf{k}, \mathbf{q})\right. \\
& +b_{\mathbf{k}+\mathbf{q}, \sigma}^{\dagger} b_{\mathbf{k},-\sigma} m_{-\mathbf{q}}^{\dagger} \Gamma_{++}(\mathbf{k}+\mathbf{q})+f_{\mathbf{k}+\mathbf{q}, \sigma}^{\dagger} f_{\mathbf{k},-\sigma} m_{-\mathbf{q}}^{\dagger} \Gamma_{+-}(\mathbf{k}, \mathbf{q})+a_{\mathbf{k}+\mathbf{q}, \sigma}^{\dagger} e_{\mathbf{k},-\sigma} n_{-\mathbf{q}}^{\dagger} \Psi_{-+}(\mathbf{k}, \mathbf{q}) \\
& +e_{\mathbf{k}+\mathbf{q}, \sigma}^{\dagger} a_{\mathbf{k},-\sigma} n_{-\mathbf{q}}^{\dagger} \Psi_{--}(\mathbf{k}, \mathbf{q})+f_{\mathbf{k}+\mathbf{q}, \sigma}^{\dagger} b_{\mathbf{k},-\sigma} n_{-\mathbf{q}}^{\dagger} \Psi_{+-}(\mathbf{k}, \mathbf{q})+b_{\mathbf{k}+\mathbf{q}, \sigma}^{\dagger} f_{\mathbf{k},-\sigma} n_{-\mathbf{q}}^{\dagger} \Psi_{++}(\mathbf{k}, \mathbf{q}) \\
& \left.+\left(a_{\mathbf{k}+\mathbf{q}, \sigma}^{\dagger} b_{\mathbf{k},-\sigma}+e_{\mathbf{k}+\mathbf{q}, \sigma}^{\dagger} f_{\mathbf{k},-\sigma}\right) m_{-\mathbf{q}}^{\dagger} \Phi_{-}(\mathbf{k}, \mathbf{q})+b_{\mathbf{k}+\mathbf{q}, \sigma}^{\dagger} a_{\mathbf{k},-\sigma}+f_{\mathbf{k}+\mathbf{q}, \sigma}^{\dagger} e_{\mathbf{k},-\sigma}\right) m_{-\mathbf{q}}^{\dagger} \Phi_{+}(\mathbf{k}, \mathbf{q}) \\
& +\left(a_{\mathbf{k}+\mathbf{q}, \sigma}^{\dagger} f_{\mathbf{k},-\sigma}+e_{\mathbf{k}+\mathbf{q}, \sigma}^{\dagger} b_{\mathbf{k},-\sigma}\right) n_{-\mathbf{q}}^{\dagger} \Xi_{-}(\mathbf{k}, \mathbf{q})+\left(f_{\mathbf{k}+\mathbf{q}, \sigma}^{\dagger} a_{\mathbf{k},-\sigma}+b_{\mathbf{k}+\mathbf{q}, \sigma}^{\dagger} e_{\mathbf{k},-\sigma}\right) n_{-\mathbf{q}}^{\dagger} \Xi_{+}(\mathbf{k}, \mathbf{q}) \\
& +h . c .] .
\end{aligned}
$$

The vertex functions in Eq. 7 are

$$
\begin{aligned}
\Phi_{ \pm}(\mathbf{k}, \mathbf{q}) & =\sqrt{2} \Delta\left[\eta_{\mathbf{q}} \pm \bar{\eta}_{\mathbf{q}}\right], \quad \Xi_{ \pm}(\mathbf{k}, \mathbf{q})=\sqrt{2} \Delta\left[\bar{\xi}_{\mathbf{q}} \pm \xi_{\mathbf{q}}\right], \\
\Gamma_{-+,--}(\mathbf{k}, \mathbf{q}) & =\frac{1}{\sqrt{2}}\left[-\eta_{\mathbf{q}}\left(\epsilon_{\mathbf{k}+\mathbf{q}}^{ \pm}+\epsilon_{\mathbf{k}}^{ \pm}\right)+\bar{\eta}_{\mathbf{q}}\left(\epsilon_{\mathbf{k}+\mathbf{q}}^{ \pm}-\epsilon_{\mathbf{k}}^{ \pm}\right)\right], \\
\Gamma_{++,+-}(\mathbf{k}, \mathbf{q}) & =\frac{1}{\sqrt{2}}\left[\eta_{\mathbf{q}}\left(\epsilon_{\mathbf{k}+\mathbf{q}}^{ \pm}+\epsilon_{\mathbf{k}}^{ \pm}\right)+\bar{\eta}_{\mathbf{q}}\left(\epsilon_{\mathbf{k}+\mathbf{q}}^{ \pm}-\epsilon_{\mathbf{k}}^{ \pm}\right)\right], \\
\Psi_{-+,--}(\mathbf{k}, \mathbf{q}) & =\frac{1}{2 \sqrt{S}}\left[-\xi_{\mathbf{q}}\left(\epsilon_{\mathbf{k}+\mathbf{q}}^{ \pm}+\epsilon_{\mathbf{k}}^{\mp}\right)+\bar{\xi}_{\mathbf{q}}\left(\epsilon_{\mathbf{k}+\mathbf{q}}^{ \pm}-\epsilon_{\mathbf{k}}^{\mp}\right)\right], \\
\Psi_{++,+-}(\mathbf{k}, \mathbf{q}) & =\frac{1}{2 \sqrt{S}}\left[\xi_{\mathbf{q}}\left(\epsilon_{\mathbf{k}+\mathbf{q}}^{ \pm}+\epsilon_{\mathbf{k}}^{\mp}\right)+\bar{\xi}_{\mathbf{q}}\left(\epsilon_{\mathbf{k}+\mathbf{q}}^{ \pm}-\epsilon_{\mathbf{k}}^{\mp}\right)\right],
\end{aligned}
$$

where, e.g. the upper signs are for $\Gamma_{-+}$and the lower ones for $\Gamma_{--}$, and

$$
\begin{array}{ll}
\eta_{\mathbf{q}}=\frac{1}{\sqrt{2}}\left(\frac{1-\nu_{\mathbf{q}}}{1+\nu_{\mathbf{q}}+J_{2} / 2 J_{1}}\right)^{1 / 4}, & \bar{\eta}_{\mathbf{q}}=\frac{1}{\sqrt{2}}\left(\frac{1+\nu_{\mathbf{q}}+J_{2} / 2 J_{1}}{1-\nu_{\mathbf{q}}}\right)^{1 / 4}, \\
\xi_{\mathbf{q}}=\frac{1}{\sqrt{2}}\left(\frac{1+\nu_{\mathbf{q}}}{1-\nu_{\mathbf{q}}+J_{2} / 2 J_{1}}\right)^{1 / 4}, \quad \bar{\xi}_{\mathbf{q}}=\frac{1}{\sqrt{2}}\left(\frac{1-\nu_{\mathbf{q}}+J_{2} / 2 J_{1}}{1+\nu_{\mathbf{q}}}\right)^{1 / 4}
\end{array}
$$

As for the case of a single-layer, the vertices which involve fermions from both, conduction and valence bands are of order $U$ whereas the vertices involving only valence or only conduction band fermions are of order $t$ and in the non-resonant regime can be omitted in the calculations to lowest order in $t / U$.

We now have all necessary tools to calculate the Raman matrix element $M_{R}$ in a diagrammatic technique. A simple experimentation shows that, just as in the case of a single layer, there are three diagrams which contribute to $M_{R}$ to leading order in $t / U$ (see Fig. 2). 
One has to keep in mind, though, that the calculation of these diagrams now involves four bands and two different type of magnons. The integration over the internal frequencies in the diagrams of Fig. 2 is straightforward, and we obtain:

1. for the diagram in Fig. 2a

$$
M_{R_{1}}^{(1,2)}= \pm 4\left(\lambda_{1,2}^{2}(\mathbf{q})+\mu_{1,2}^{2}(\mathbf{q})\right) \frac{1}{N} \sum_{\mathbf{k}, \alpha, \beta}^{\prime}\left(\frac{\partial \epsilon_{\mathbf{k}}}{\partial k_{\alpha}} e_{i \alpha}\right)\left(\frac{\partial \epsilon_{\mathbf{k}-\mathbf{q}}}{\partial k_{\beta}} e_{f \beta}\right) \frac{4 \Delta}{4 \Delta^{2}-\Omega^{2}}
$$

where the upper sign is for $M_{R_{1}}^{(1)}$, and the lower sign for $M_{R_{1}}^{(2)}$, the indices 1 and 2 indicate whether the final state contains two magnons of type $m_{\mathbf{q}}$ or of type $n_{\mathbf{q}}$, respectively, and $\Omega$ is a frequency equal to $\omega_{i}$ or $\omega_{f}$ which are indistinguishable to leading order in $t / U$;

2. for the diagram in Fig. $2 \mathrm{~b}$

$$
M_{R_{2}}^{(1,2)}=-4 \lambda_{1,2}(\mathbf{q}) \mu_{1,2}(\mathbf{q}) \frac{1}{N_{\mathbf{k}, \alpha, \beta}} \sum^{\prime}\left(\frac{\partial \epsilon_{\mathbf{k}}}{\partial k_{\alpha}} e_{i \alpha}\right)\left(\frac{\partial \epsilon_{\mathbf{k}}}{\partial k_{\beta}} e_{f \beta}\right)\left[\frac{8 \Delta}{4 \Delta^{2}-\Omega^{2}}+\frac{8 \Delta\left(4 \Delta^{2}+\Omega^{2}\right)}{\left(4 \Delta^{2}-\Omega^{2}\right)^{2}}\right] ;
$$

3. for the diagram in Fig. 20

$$
M_{R_{3}}^{(1,2)}=4 \lambda_{1,2}(\mathbf{q}) \mu_{1,2}(\mathbf{q}) \frac{1}{N} \sum_{\mathbf{k}, \alpha, \beta}^{\prime}\left(\frac{\partial \epsilon_{\mathbf{k}}}{\partial k_{\alpha}} e_{i \alpha}\right)\left(\frac{\partial \epsilon_{\mathbf{k}}}{\partial k_{\beta}} e_{f \beta}\right) \frac{8 \Delta\left(4 \Delta^{2}+\Omega^{2}\right)}{\left(4 \Delta^{2}-\Omega^{2}\right)^{2}} .
$$

As before, $\mathbf{e}_{i, f}$ are the polarization vectors of the incident and scattered photons, respectively, and the coefficients $\mu_{1,2}(\mathbf{q})$ and $\lambda_{1,2}(\mathbf{q})$ are defined as

$$
\begin{array}{ll}
\sqrt{2} \mu_{1}(\mathbf{q})=\bar{\eta}_{\mathbf{q}}+\eta_{\mathbf{q}}, & \sqrt{2} \lambda_{1}(\mathbf{q})=\bar{\eta}_{\mathbf{q}}-\eta_{\mathbf{q}} \\
\sqrt{2} \mu_{2}(\mathbf{q})=\bar{\xi}_{\mathbf{q}}+\xi_{\mathbf{q}}, & \sqrt{2} \lambda_{2}(\mathbf{q})=\bar{\xi}_{\mathbf{q}}-\xi_{\mathbf{q}} .
\end{array}
$$

Simple algebra yields the relations

$$
\mu_{1,2}(\mathbf{q})=\left[\frac{1}{2}\left(\frac{4 J_{1}+J_{2}}{2 \Omega_{1,2}(\mathbf{q})}+1\right)\right]^{1 / 2}, \quad \lambda_{1,2}(\mathbf{q})=\frac{4 J_{1} \nu_{\mathbf{q}} \pm J_{2}}{\left|4 J_{1} \nu_{\mathbf{q}} \pm J_{2}\right|}\left[\frac{1}{2}\left(\frac{4 J_{1}+J_{2}}{2 \Omega_{1,2}(\mathbf{q})}-1\right)\right]^{1 / 2} .
$$

Comparing these results with those in Ref. [12] we observe that the form of Eqs. (10)-(12) is the same for a single- and a double-layer system. The information about the coupling 
between the layers is therefore only contained in the coherence factors $\mu_{1,2}(\mathbf{q})$ and $\lambda_{1,2}(\mathbf{q})$. This is a direct consequence of the fact that the photons' vector potential only couples to the in-plane fermionic current.

Finally, we use the relations

$$
\begin{aligned}
\frac{1}{N} \sum_{\mathbf{k}, \alpha, \beta}^{\prime}\left(\frac{\partial \epsilon_{\mathbf{k}}}{\partial k_{\alpha}} e_{i \alpha}\right)\left(\frac{\partial \epsilon_{\mathbf{k}-\mathbf{q}}}{\partial k_{\beta}} e_{f \beta}\right) & =t^{2}\left[e_{i x} e_{f x} \cos q_{x}+e_{i y} e_{f y} \cos q_{y}\right] \\
\frac{1}{N} \sum_{\mathbf{k}, \alpha, \beta}^{\prime}\left(\frac{\partial \epsilon_{\mathbf{k}}}{\partial k_{\alpha}} e_{i \alpha}\right)\left(\frac{\partial \epsilon_{\mathbf{k}}}{\partial k_{\beta}} e_{f \beta}\right) & =t^{2}\left[e_{i x} e_{f x}+e_{i y} e_{f y}\right]
\end{aligned}
$$

substitute them into Eqs. (10)-(12), and obtain for the total Raman matrix element $M_{R}^{(1,2)}=$

$$
\begin{aligned}
& M_{R_{1}}^{(1,2)}+M_{R_{2}}^{(1,2)}+M_{R_{3}}^{(1,2)} \\
& M_{R}^{(1,2)}=-8 t^{2} \frac{2 \Delta}{4 \Delta^{2}-\Omega^{2}}\left[2 \lambda_{1,2}(\mathbf{q}) \mu_{1,2}(\mathbf{q})\left(e_{i x} e_{f x}+e_{i y} e_{f y}\right) \mp\right. \\
&\left.\left(\lambda_{1,2}^{2}(\mathbf{q})+\mu_{1,2}^{2}(\mathbf{q})\right)\left(e_{i x} e_{f x} \cos q_{x}+e_{i y} e_{f y} \cos q_{y}\right)\right] .
\end{aligned}
$$

Notice that $M_{R}^{(1)}(\mathbf{q})=M_{R}^{(2)}(\mathbf{q}+\mathbf{Q})$. Since for the calculation of the Raman intensity we have to integrate over the whole Brillouin zone we can restrict our consideration to only one type of magnons, and just multiply $M_{R}$ by $\sqrt{2}$.

We now change tracks and compute the matrix element $M_{R}$ within the Loudon-Fleury theory [16], i.e. we assume that the spins interact via the Heisenberg Hamiltonian

$$
H=J_{1} \sum_{<i, j>, \alpha} \mathbf{S}_{\alpha, i} \cdot \mathbf{S}_{\alpha, j}+J_{2} \sum_{i} \mathbf{S}_{1, i} \cdot \mathbf{S}_{2, i}
$$

As before, $\alpha=1,2$, and the scattering of light is described by the Loudon-Fleury Hamiltonian

$$
H_{L-F}=\Lambda \sum_{j, \delta, \alpha}\left(e_{i x} e_{f x} \mathbf{S}_{j, \alpha} \cdot \mathbf{S}_{j+\delta_{x}, \alpha}+e_{i y} e_{f y} \mathbf{S}_{j, \alpha} \cdot \mathbf{S}_{j+\delta_{y}, \alpha}\right)
$$

Here, $\Lambda$ is a coupling constant and $\delta=\left(\delta_{x}, \delta_{y}\right)$ is a vector to nearest neighbors sites in a plane. Observe that this scattering Hamiltonian has the same form as for a single layer. This is again a consequence of the fact that the light only couples to the in-plane fermionic current. Following the standard procedure, the spin operators are now transformed to 
boson operators via the conventional Holstein-Primakoff or Dyson-Maleev expansions. The easiest way to proceed is to introduce just one Bose field with momentum in the full first Brillouin zone, and then perform a unitary rotation to magnon operators which diagonalize the quadratic form for a two-layer Heisenberg antiferromagnet. As for a single plane, the transformation to magnon operators involves the same coefficients $\lambda_{1}(\mathbf{q})$ and $\mu_{1}(\mathbf{q})$ as in Eq. (14).

Retaining only the term in the scattering Hamiltonian which contains two magnon creation operators, we obtain for the Loudon-Fleury matrix element

$$
\begin{aligned}
& M_{R_{L F}}=- \Lambda\left[2 \lambda_{1}(\mathbf{q}) \mu_{1}(\mathbf{q})\left(e_{i x} e_{f x}+e_{i y} e_{f y}\right)-\right. \\
&\left.\left(\lambda_{1}^{2}(\mathbf{q})+\mu_{1}^{2}(\mathbf{q})\right)\left(e_{i x} e_{f x} \cos q_{x}+e_{i y} e_{f y} \cos q_{y}\right)\right] .
\end{aligned}
$$

Comparing the two expressions for $M_{R}$, Eq. 16 and Eq. 19, we see that they are identical provided we identify the coupling constant

$$
\Lambda=8 \sqrt{2} t^{2}\left[\frac{2 \Delta}{4 \Delta^{2}-\Omega^{2}}\right]
$$

This concludes our derivation of the Loudon-Fleury Hamiltonian for a two-layer system.

Before we proceed with the calculations of the Raman intensity, we would like to comment on the form of the Loudon-Fleury Hamiltonian. In some phenomenological theories for a single layer, the interaction Hamiltonian between light and spin degrees of freedom is written as

$$
H_{L F}=\Lambda \sum_{j, \delta} P\left(\mathbf{e}_{i}, \mathbf{e}_{f}, \delta\right) \mathbf{S}_{\alpha, j} \cdot \mathbf{S}_{\alpha, j+\delta}
$$

where

$$
P\left(\mathbf{e}_{i}, \mathbf{e}_{f}, \delta\right)=\left[\frac{1}{2} \mathbf{e}_{i} \cdot \mathbf{e}_{f}-\left(\delta \cdot \mathbf{e}_{i}\right)\left(\delta \cdot \mathbf{e}_{f}\right)\right]
$$

This formula is obtained from (18) if the term which describes scattering in $A_{1 g}$ geometry is neglected. For a single layer, this procedure is legitimate as the scattering Hamiltonian in $A_{1 g}$ geometry commutes with the Heisenberg Hamiltonian, and consequently there is no $A_{1 g}$ 
scattering. However, for a two-layer system, more care is needed as the Heisenberg Hamiltonian now contains an extra term with an interlayer coupling, which does not commute with $H_{L F}$. As a result, if we want to rewrite the Loudon-Fleury Hamiltonian for two layers using the projection operator $P$, we necessarily have to introduce an extra term which contains spins from two different planes. Specifically, the Loudon-Fleury Hamiltonian expressed in terms of projection operators should have the form

$$
H_{L F}=\Lambda_{B 1} \sum_{j, \delta, \alpha} P\left(\mathbf{e}_{i}, \mathbf{e}_{f}, \delta\right) \mathbf{S}_{\alpha, j} \cdot \mathbf{S}_{\alpha, j+\delta}+\Lambda_{A 1} \sum_{j} P\left(\mathbf{e}_{i}, \mathbf{e}_{f}, 0\right) \mathbf{S}_{1, j} \cdot \mathbf{S}_{2, j}
$$

where $P\left(\mathbf{e}_{i}, \mathbf{e}_{f}, \delta\right)$ is the same as before. Here, the first term describes scattering in $B_{1 g}$ geometry, while the second term (which couples spins from different layers) contributes to $A_{1 g}$ scattering. Comparing the two forms of the Loudon-Fleury Hamiltonians, we obtain for the two coupling constants

$$
\Lambda_{A 1}=4 t^{2}\left[\frac{2 \Delta}{4 \Delta^{2}-\Omega^{2}}\right] \frac{J_{2}}{J_{1}}, \quad \Lambda_{B 1}=-8 t^{2}\left[\frac{2 \Delta}{4 \Delta^{2}-\Omega^{2}}\right]
$$

The two forms of the Loudon-Fleury Hamiltonian indeed yield the same Raman matrix element as in Eq. (19).

\section{THE RAMAN INTENSITY IN THE NON-RESONANT REGIME}

\section{A. The Non-Interacting Case}

The Raman scattering cross section is proportional to the Golden Rule transition rate 29

$$
R=\frac{8 \pi^{3} e^{4}}{\hbar^{3} V^{2} \omega_{i} \omega_{f}} \sum\left|M_{R}\right|^{2} \delta\left(\hbar \omega_{i}-\hbar \omega_{f}+\epsilon_{i}-\epsilon_{f}\right)
$$

where $i$ and $f$ are the initial and final states of the system, $\epsilon_{i, f}$ are the corresponding energies, and the summation runs over all possible initial and final electronic states. Let us first neglect final state magnon-magnon interaction. Then, $\epsilon_{i}-\epsilon_{f}=2 \Omega_{1}(\mathbf{q})$, and using 
(16) we obtain for the Raman intensity in the $A_{1 g}$ and $B_{1 g}$ channels (dropping an identical overall prefactor)

$$
\begin{aligned}
I_{A_{1 g}}(\Omega) & \propto 2 \sum_{\mathbf{q}}\left(M_{R}^{(1)}\right)^{2} \delta\left(\hbar \omega_{i}-\hbar \omega_{f}-2 \Omega_{1}(\mathbf{q})\right) \\
& =2 \Lambda^{2} \sum_{\mathbf{q}}\left(\frac{J_{2}}{4 J_{1}}\right)^{2}\left(\frac{2 J_{1}\left(1-\nu_{\mathbf{q}}\right)}{\Omega_{1}(\mathbf{q})}\right)^{2} \delta\left(\hbar \omega_{i}-\hbar \omega_{f}-2 \Omega_{1}(\mathbf{q})\right) \\
I_{B_{1 g}}(\Omega) & \propto 2 \sum_{\mathbf{q}}\left(M_{R}^{(1)}\right)^{2} \delta\left(\hbar \omega_{i}-\hbar \omega_{f}-2 \Omega_{1}(\mathbf{q})\right) \\
& =2 \Lambda^{2}\left(1+\frac{J_{2}}{4 J_{1}}\right)^{2} \sum_{\mathbf{q}}\left(\frac{2 J_{1} \tilde{\nu}_{\mathbf{q}}}{\Omega_{1}(\mathbf{q})}\right)^{2} \delta\left(\hbar \omega_{i}-\hbar \omega_{f}-2 \Omega_{1}(\mathbf{q})\right)
\end{aligned}
$$

where $\Omega=\omega_{i}-\omega_{f}$ and we have defined $\tilde{\nu}_{\mathbf{q}}=\left(\cos q_{x}-\cos q_{y}\right) / 2$. We see that the Raman intensity in the $A_{1 g}$ channel is proportional to $\left(J_{2} / J_{1}\right)^{2}$ and thus vanishes with vanishing $J_{2}$. In $B_{1 g}$ geometry, the changes in $I_{B_{1 g}}$ imposed by the interlayer coupling are minor: the form of the matrix element is preserved and the only changes appear in the prefactor and in the magnon energy dispersion $\Omega_{1}(\mathbf{q})$. The momentum sums in Eq. (26) can be conveniently reduced to complete elliptic integrals. The resulting expressions for the intensities are, however, rather involved; they are collected in Appendix Q. Here we discuss only the main features of the Raman spectrum.

Our key observation is the following: The magnon energy $\Omega_{1}(\mathbf{q})$ is gapless at the zone center $\mathbf{q}=0$, and has a gap, $\Omega_{1}(\mathbf{Q})=2 \sqrt{J_{2} J_{1}}$ at $\mathbf{q}=\mathbf{Q}$. Then, for $\Omega=\omega_{i}-\omega_{f}<2 \Omega_{1}(\mathbf{Q})$, only magnons with momentum near $\mathbf{q} \approx 0$ can be excited. The numerators in both scattering geometries vanish at $\mathbf{q}=0$, and it is not difficult to show that the contributions from the $\mathbf{q} \approx 0$ region yield $I(\Omega) \propto \Omega^{3}$ (see also below). For $\Omega>2 \Omega_{1}(\mathbf{Q})$, however, also magnons with $\mathbf{q} \approx \mathbf{Q}$ can be excited. In $B_{1 g}$ geometry, the numerator in $I_{B_{1 g}}$ contains the factor $\tilde{\nu}_{\mathbf{q}}^{2}$ which vanishes at $\mathbf{q}=\mathbf{Q}$ such that the opening of a new scattering channel does not cause substantial changes in the intensity which still scales as $\Omega^{3}$. However, in $A_{1 g}$ geometry the numerator in $I_{A_{1 g}}$ at $\mathbf{q}=\mathbf{Q}$ is just a positive constant. In this situation, the scattering intensity changes drastically at $\Omega=2 \Omega_{1}(\mathbf{Q})$ : using Eq. (26), we find

$$
\Delta I_{r e l}=\frac{I_{A_{1 g}}\left(2 \Omega_{1}(\mathbf{Q})+\delta \omega\right)-I_{A_{1 g}}\left(2 \Omega_{1}(\mathbf{Q})-\delta \omega\right)}{I_{A_{1 g}}\left(2 \Omega_{1}(\mathbf{Q})-\delta \omega\right)}=\frac{256 J_{1}^{4}}{\left(\Omega_{1}(\mathbf{Q})\right)^{4}} .
$$


For $J_{2} / J_{1}=0.1$ we obtain $\Delta I_{\text {rel }} \sim 1600$, i.e. the enhancement of the $A_{1 g}$ signal at the threshold frequency is very strong.

Moreover, the value of the intensity in the $A_{1 g}$ channel right above the threshold, $I_{A_{1 g}} \propto$ $J_{2}^{2} / \Omega_{1}(\mathbf{Q}) \propto J_{1}\left(J_{2} / J_{1}\right)^{3 / 2}$, has the same order of magnitude as the intensity in the $B_{1 g}$ channel, $I_{B_{1 g}} \propto\left(\Omega_{1}(\mathbf{Q})\right)^{3} \propto\left(J_{2} / J_{1}\right)^{3 / 2}$. We found that for all reasonable values for $J_{2} / J_{1}$ the ratio of intensities is $\sim 1.7-1.9$. In other words, if the Loudon-Fleury approximation is applicable, and if one claims to observe the two-magnon profile in $B_{1 g}$ geometry at around $\Omega_{1}(\mathbf{Q})$, one should also observe, in a two-layer system, the signal of an even larger intensity in the $A_{1 g}$ geometry.

The intensities for the $A_{1 g}$ and $B_{1 g}$ geometries without final state interaction are plotted in Fig. 3 for two different values of $J_{2} / J_{1}$. There are unphysical singularities in both intensities at the maximum magnon energy, but just as in the case of a single layer, they are artifacts of neglecting interactions between magnons. We will see in Sec. IIIB that once an interaction is included, the unphysical singularities are removed.

\section{B. The Interacting Case}

We now analyze how the two-magnon profile changes when the interaction between magnons is included. Fist of all, the magnon-magnon interaction renormalizes the spinwave spectrum. To leading order in $1 / S$, which we only consider here, this renormalization can be absorbed into the renormalization of the exchange couplings

$$
\begin{array}{ll}
J_{1} \rightarrow J_{1}\left(1+\frac{r}{2 S}\right), & r=1-\frac{1}{N} \sum_{\mathbf{q}} \frac{\left(1-\nu_{\mathbf{q}}\right)\left(4 J_{1}\left(1+\nu_{\mathbf{q}}\right)+J_{2}\right)}{2 \Omega_{1}(\mathbf{q})} \\
J_{2} \rightarrow J_{2}\left(1+\frac{r^{\prime}}{2 S}\right), & r^{\prime}=1-\frac{1}{N} \sum_{\mathbf{q}} \frac{4 J_{1}\left(1-\nu_{\mathbf{q}}\right)}{2 \Omega_{1}(\mathbf{q})} .
\end{array}
$$

where the momentum sums run over the whole first Brillouin zone (notice that we defined $\Omega_{1}$ without a factor of $2 S$ ). This renormalization comes from one-loop diagrams (Oguchi corrections [30]). Beyond the leading order in $1 / S$, one has to solve Eq. (28) self-consistently and also include corrections with higher number of loops. Numerically, however, it turns out 
that the dominant correction, at least to order $1 / S^{2}$, still comes from one-loop diagrams 31. In other words, the actual magnon dispersion nearly preserves the same form as in linear spin wave theory, but contains renormalized coupling constants $J_{1,2}$. Below we will assume that this renormalization is already included into the definitions of $J_{1,2}$ and neglect it in our further consideration.

Strictly speaking, to justify this approximation for the calculation of the effects due to a final state magnon-magnon interaction, we also have to prove that the dominant renormalization of the four-magnon interaction vertex can be absorbed into the same renormalization of the exchange integrals. We did not perform explicit $1 / S$ calculations for the vertex. However, as the vertex itself has a factor $1 / S$ in comparison to the magnon frequency, and all calculations involving magnon-magnon scattering will be performed only to the leading order in $1 / S$, then whether to use a bare or a renormalized $J_{1,2}$ in the vertex is beyond the accuracy of our calculations. For simplicity, we will henceforth use renormalized values of $J_{1,2}$ everywhere.

We now consider in detail the renormalization of the two-magnon profile due to multiple scattering of two magnons. The magnon-magnon vertices can be immediately obtained from the Heisenberg Hamiltonian by applying e.g. the Holstein-Primakoff transformation to boson operators and a subsequent canonical transformation to magnon operators which diagonalize the quadratic part of the spin wave Hamiltonian. A detailed study of the effects due to magnon-magnon interaction in a single-layer antiferromagnet was already performed by Canali and Girvin [11], and we follow here their line of reasoning. To leading order in $1 / S$, we can restrict ourselves to the scattering process which conserves the number of magnons. The effective scattering Hamiltonian then takes the form

$$
H_{m a g-m a g}=\frac{1}{N} \sum_{\mathbf{k}, \mathbf{q}} V(\mathbf{k}, \mathbf{q}) \alpha_{\mathbf{q}}^{\dagger} \beta_{-\mathbf{q}}^{\dagger} \alpha_{\mathbf{k}} \beta_{-\mathbf{k}}
$$

with

$$
V(\mathbf{k}, \mathbf{q})=A \frac{B_{\mathbf{k}} B_{\mathbf{q}}}{\Omega_{1}(\mathbf{k}) \Omega_{1}(\mathbf{q})}-B_{\mathbf{q}-\mathbf{k}}\left[\frac{A^{2}}{\Omega_{1}(\mathbf{k}) \Omega_{1}(\mathbf{q})}+1\right]
$$


and

$$
A=\left(J_{2}+4 J_{1}\right) / 2, \quad B_{\mathbf{k}}=\left(J_{2}+4 J_{1} \nu_{\mathbf{k}}\right) / 2 .
$$

In terms of $A$ and $B_{\mathbf{k}}$ the magnon dispersion is given by $\Omega_{1}(\mathbf{k})=\sqrt{A^{2}-B_{\mathbf{k}}^{2}}$.

In order to find the full vertex function for repeated two-magnon scattering we need to sum an infinite series of ladder diagrams. In $B_{1 g}$ geometry, the "side" vertices from the electron-photon coupling scale as $\tilde{\gamma}_{\mathbf{q}}=\left(\cos q_{x}-\cos q_{y}\right) / 2$, and it is easy to see that the only term in Eq. (30) which contributes to scattering is the one with $\nu_{\mathbf{q}-\mathbf{k}}$. The evaluation of the ladder diagram series then proceeds exactly in the same way as for a single-layer system [11,32. The analytical solution is presented in Appendix D.

The plots for the Raman intensity $I_{B_{1 g}}$ for two values of $J_{2} / J_{1}$ are shown in Fig. $甘$. The unphysical singularity that we found in the non-interacting case disappears, as expected, and we observe a pronounced two-magnon peak. We see that with increasing $J_{2} / J_{1}$ the two-magnon peak not only shifts to higher frequencies but that the amplitude of the signal also slightly increases. The latter, however, is mainly due to the overall factor $\left(1+J_{2} / 4 J_{1}\right)$ in the matrix element and a renormalization of the Loudon-Fleury constant $\Lambda$ from magnonmagnon interactions. The shift of the peak position towards higher frequencies can be understood in the simple picture that the incoming photon flips two neighboring spins on the same layer. This creates misaligned spin pairs and thereby increases the total energy of the system. Evaluating the corresponding energy increase for a Néel state, we obtain a two-magnon peak at $\Omega=3 J_{1}\left(1+J_{2} / 3 J_{1}\right)$ which is roughly consistent with what we find.

In $A_{1 g}$ geometry, the solution of the ladder series is more difficult since the "side" vertex behaves as $\sim\left(1-\nu_{\mathbf{q}}\right) / \Omega_{1}(\mathbf{q})$ where $\mathbf{q}$ is the magnon momentum. At small $\mathbf{q}$, this vertex scales linearly with $\mathbf{q}$ as a consequence of the Adler principle [33]: the Raman matrix element includes the interaction between fermions and Goldstone bosons, and this interaction should vanish at the points where the magnon energy turns to zero. Because of the extra power of momentum in $M_{R}$, the Raman intensity without final state interaction scales as $I_{A_{1 g}} \propto \Omega^{3}$ at very low frequencies, as mentioned before. However, the form of the "side" vertex in $A_{1 g}$ 
geometry, is not reproduced at the magnon-magnon vertex, and we in fact have to solve a set of coupled integral equations in order to get the result for the full $I_{A_{1 g}}$. The explicit expression for $I_{A_{1 g}}$ is rather cumbersome, so we present it in Appendix $\mathrm{D}$ and here discuss only the key features of the solution.

As the "side" vertices for $A_{1 g}$ are invariant under transformations of the symmetry group $D_{4 h}$ of the square lattice, we can restrict ourselves to only that part of the scattering potential $V(\mathbf{k}, \mathbf{q})$, which has the same symmetry, i.e.

$$
V(\mathbf{k}, \mathbf{q})=-2 J_{1}\left[\nu_{\mathbf{k}} \nu_{\mathbf{q}}+\frac{J_{2}}{4 J_{1}}+\frac{J_{2}}{4 J_{1}}\left(1+\frac{J_{2}}{4 J_{1}}\right) \frac{\left(2 J_{1}\right)^{2}\left(1-\nu_{\mathbf{k}}\right)\left(1-\nu_{\mathbf{q}}\right)}{\Omega_{\mathbf{q}} \Omega_{\mathbf{k}}}\right] .
$$

We see that $V(\mathbf{k}, \mathbf{q})$ actually tends to a finite value for $\mathbf{k}=\mathbf{q}=0$. The cubic frequency dependence of $I_{A_{1 g}}(\Omega)$ is therefore actually an artifact of neglecting the final state interaction. When this interaction is included, $I_{A_{1 g}}$ scales linearly with $\Omega$ at the lowest frequencies. We also found that the real part of the polarizability has a logarithmic singularity at $\Omega=$ $2 \Omega_{1}(\mathbf{Q})$. This singularity gives rise to two effects: first, it makes $I_{A_{1 g}}$ a continuous function of frequency, in other words, eliminates a jump in the intensity at $2 \Omega_{1}(\mathbf{Q})$. Second, it gives rise to a strong peak in $I_{A_{1 g}}$ at frequencies somewhat smaller than $2 \Omega_{1}(\mathbf{Q})$. Specifically, we found that near $2 \Omega_{1}(\mathbf{Q})$, the dominant contribution to the intensity comes from the third term in Eq. (32), and $I_{A_{1 g}}$ has the form

$$
I_{A_{1 g}} \propto J_{2}^{2} \frac{\Omega_{1}^{3}(\mathbf{Q})}{\left(1+R_{1}\right)^{2}+R_{2}^{2}}+I_{A_{1 G}}^{\prime}
$$

where

$$
R_{1}=\frac{J_{2}}{2 \pi \Omega_{1}(\mathbf{Q})} \ln \frac{2 \Omega_{1}(\mathbf{Q})-\Omega}{2 \Omega_{1}(\mathbf{Q})} \quad, \quad R_{2}=\frac{1}{4} \frac{J_{2}}{J_{1}}\left(\frac{\Omega_{1}(\mathbf{Q})}{2 J_{1}}\right)^{3} .
$$

$I_{A_{1 g}}^{\prime}$ remains finite at $\Omega=2 \Omega_{1}(\mathbf{Q})$ and is proportional to $J_{2}^{2} / \Omega_{1}(\mathbf{Q})$. We see that there exists a very narrow peak in $I_{A_{1 g}}$ located at $\Omega=2 \Omega_{1}(\mathbf{Q})\left(1-\exp \left(-2 \pi \Omega_{1}(\mathbf{Q}) / J_{2}\right)\right)$. The intensity right at the peak is very high, $I_{A_{1 g}} \propto 1 / \Omega_{1}^{3}(\mathbf{Q})$. At small $J_{2}$, the peak position is exponentially close to $2 \Omega_{1}(\mathbf{Q})$. However, at larger $J_{2}$, we found numerically that the peak is actually located at frequencies significantly smaller than $2 \Omega_{1}(\mathbf{Q})$. This last result agrees with the calculations of the two-magnon absorption profile in Ref. [20]. 
The solutions for $I_{A_{1 g}}$ for two different values of $J_{2} / J_{1}$ are graphically presented in Fig. 5 . The $A_{1 g}$ Raman spectra have been evaluated on a finite lattice with $1000 \times 1000$ lattice points. A finite imaginary part $i \delta$ has been added to the energy denominators of the spin wave propagators in Eqs. (D6), (D7), and (D8) in Appendix D. This allows to study the influence of damping on the $A_{1 g}$ two-magnon spectra. Without damping, the imaginary part of the polarizability has a jump at $\Omega=2 \Omega_{1}(\mathbf{Q})$, and by the Kramers-Kronig relation, the real part of polarizabiliy $\left(R_{1}\right.$ term in Eq. (34)) necessarily has a logarithmic singularity, see Fig. 5c. With damping, the singular behavior near the threshold frequency is removed and the peak position is shifted closer to $2 \Omega_{1}(\mathbf{Q})$. Note also that, as in $B_{1 g}$ geometry, the divergence at twice the maximum spin wave frequency is removed due to the final state magnon-magnon interaction.

Finally, for the purpose of comparison with experiments, it is useful to compute the ratio of the Raman intensities for $A_{1 g}$ and $B_{1 g}$ geometries right at their peak positions. We found that this ratio is actually very small: for $J_{2} / J_{1}=0.1$ it is about 0.009 whereas for $J_{2} / J_{1}=0.3$, it is 0.044 . In other words, though the $A_{1 g}$ intensity at the peak is larger than the intensity of the $B_{1 g}$ signal at the same frequency, the overall scale of the peak is only a few percent of the two-magnon peak in $B_{1 g}$ geometry. We therefore have to conclude that in the non-resonant regime where the Loudon-Fleury theory is applicable, the extra peak in $A_{1 g}$ geometry can hardly be separated from the background signal. We now consider what happens in the resonant regime, i.e. when the incident photon frequency becomes comparable to the Mott-Hubbard gap.

\section{THE RAMAN INTENSITY IN THE RESONANT REGIME}

In our derivation of the Loudon-Fleury Hamiltonian, we have chosen the diagrams to leading order in $t / U$ under the assumption that the energy of the incoming and outgoing photons is much smaller than the energy gap between the conduction and valence bands. Under these conditions, all denominators in the diagrams Figs. 2a-c were of order $U$ which 
in turn allowed us to omit all diagrams with intraband scattering. The situation, however, becomes different in the resonant regime where the energy of the incoming photon comes close to the Mott-Hubbard gap $2 \Delta \sim U$. Actually, most of the experiments on two-magnon Raman scattering in parent high- $T_{c}$ compounds have been done with visible light frequencies which are only $O(J)$ apart from $2 \Delta$.

It was shown in Ref. 12] that in the resonant regime, the diagrams with intraband scattering are more relevant than those which contribute to the Loudon-Fleury theory, and, moreover, the dominant contribution to Raman scattering comes from just one diagram shown schematically in Fig. 6. This diagram yields a Raman matrix element $M_{R}^{t r}=M_{R}^{+}+$ $M_{R}^{-}$, where

$$
M_{R}^{ \pm}=8 i \frac{1}{N} \sum_{\mathbf{k}, \alpha, \beta}^{\prime} \frac{\left(\frac{\partial \epsilon_{\mathbf{k}}}{\partial k_{\alpha}} e_{i \alpha}\right)\left(\frac{\partial \epsilon_{\mathbf{k}-\mathbf{q}}}{\partial k_{\beta}} e_{f \beta}\right)\left[\mu_{\mathbf{q}} \epsilon_{\mathbf{k}-\mathbf{q}}^{ \pm}-\lambda_{\mathbf{q}} \epsilon_{\mathbf{k}}^{ \pm}\right]^{2}}{\left(\omega_{i}-2 E_{\mathbf{k}}^{ \pm}+i \delta\right)\left(\omega_{i}-\Omega_{1}(\mathbf{q})-E_{\mathbf{k}}^{ \pm}-E_{\mathbf{k}-\mathbf{q}}^{ \pm}+i \delta\right)\left(\omega_{f}-2 E_{\mathbf{k}-\mathbf{q}}^{ \pm}+i \delta\right)} .
$$

One of the key consequences of considering the resonance regime is that there exists a nonzero signal in $A_{1 g}$ geometry even for a single-layer. Indeed, the absence of the $A_{1 g}$ signal in the Loudon-Fleury theory was related to a particular form of the interaction Hamiltonian $H_{L F}$ which contained only spin degrees of freedom. The inclusion of the intraband processes modifies the form of the interaction Hamiltonian with light, in which case it no longer commutes with the Heisenberg Hamiltonian even when $\mathbf{e}_{i}=\mathbf{e}_{f}=(\hat{x}+\hat{y}) / \sqrt{2}$.

The feature of the diagram in Fig. 6 which makes it dominant for $B_{1 g}$ scattering in the resonance regime is that it allows all three denominators to vanish simultaneously leading to a triple resonance enhancement [12]. For two-layer systems, we should check whether or not the rapid variation of $I_{A_{1 g}}$ near $2 \Omega_{1}(\mathbf{Q})$, can be enhanced when the incident photon frequency is tuned right to the triple resonance value. We performed computations analogous to those in [12] and found that there is in fact no enhancement at the frequency threshold for $A_{1 g}$ scattering because the occurrence of the triple resonance requires that the fermionic velocities at momenta $\mathbf{k}_{0}\left(\omega_{i}=2 E^{ \pm}\left(\mathbf{k}_{0}\right)\right)$ and $\mathbf{k}_{0}+\mathbf{q}$, where $\mathbf{q}$ is the magnon momentum, be antiparallel to each other. For $\mathbf{q}=\mathbf{Q}$, we evidently have $\left.\nabla_{\mathbf{k}} E_{\mathbf{k}}^{ \pm}\right|_{\mathbf{k}_{0}}=\left.\nabla_{\mathbf{k}} E_{\mathbf{k}}^{ \pm}\right|_{\mathbf{k}_{0}+\mathbf{Q}}$, i.e. the 
two velocities are parallel. In this situation, the integration over the fermionic momenta near $\mathbf{k}_{0}$ gives zero because all poles lie in the same half-plane. We also performed more detailed calculations by expanding the denominators up to second order around $\mathbf{k}_{0}$. This actually makes the integral over $\mathbf{k}-\mathbf{k}_{0}$ finite, but still there is no singularity in $M_{R}^{t r}$ at $\omega_{i}-2 E_{\mathbf{k}_{0}}$, so we do not expect any substantial enhancement of the Raman intensity in the $A_{1 g}$ channel due to a triple resonance.

Despite the absence of the enhancement, the diagram in Fig. 6 is still relevant in the resonant regime simply because it contains three denominators which all are $O(J)$. Since there is no resonant enhancement, then, to first approximation, one can just set the denominator in (35) to a constant and consider the basic structure of $M_{R}$ as imposed by the interaction vertices between magnons and fermions. Performing simple calculations, we obtained from (35)

$$
M_{R}^{t r} \propto\left(\nu_{\mathbf{q}} \Omega_{1}(\mathbf{q})+\frac{J_{1} J_{2}}{4} \frac{1-\nu_{\mathbf{q}}}{\Omega_{1}(\mathbf{q})}\right) .
$$

At small frequencies, the contributions to the Raman intensity come only from magnon momenta near $\mathbf{q}=0$. We see from (36) that for these momenta, $M_{R}^{t r}$ scales linearly with the magnon momentum, just as we found in the Loudon-Fleury approximation. Clearly then, the full Raman intensity in the absence of magnon-magnon interaction scales as $I_{A_{1 g}} \propto \Omega^{3}$ at small frequencies. We studied the effects of the magnon-magnon interaction and found that, as before, the bare form of the side vertex is not reproduced in a perturbation theory for magnon-magnon scattering, and the finite state interaction gives rise to a linear, rather than cubic frequency dependence of $I_{A_{1 g}}$. Moreover, as $M_{R}^{t r}$ does not contain $J_{2}$ as the overall factor, it obviously gives a dominant contribution to $I_{A_{1 g}}$. This in turn implies that at $\Omega<2 \Omega_{1}(\mathbf{Q})$, the Raman intensity in a double-layer system is roughly half of the intensity in a one-layer system.

For $\Omega>2 \Omega_{1}(\mathbf{Q})$, magnon momenta near $\mathbf{q}=\mathbf{Q}$ also contribute to $I(\Omega)$. It is not difficult to verify that this extra contribution has the same dependence on $J_{2} / J_{1}$ as in the Loudon-Fleury theory. Accordingly, if we consider only $M_{R}^{t r}$, we obtain qualitatively the 
same form of the $A_{1 g}$ intensity profile as in the Loudon-Fleury theory - the only difference is that now the intensities below and above the jump at $2 \Omega_{1}(\mathbf{Q})$ are of the same order of magnitude. Our result for the $A_{1 g}$ intensity computed with $M_{R}^{t r}$ with final state interaction is presented in Fig. 7. Qualitatively, the intensity profile is the same as in the LoudonFleury approximation, but the new features are a substantial increase in the intensity above the threshold at $2 \Omega_{1}(\mathbf{Q})$ and the flattening of the $A_{1 g}$ intensity slightly above the threshold frequency. There may also be a very narrow peak slightly below the threshold frequency (just as we obtained in the Loudon-Fleury theory), which we do not see because of a limited numerical accuracy. In any event, however, the singular behavior at this peak is eliminated by damping.

The total matrix element for the $A_{1 g}$ scattering is a sum of Loudon-Fleury and triple resonance contributions. Without studying in detail the frequency dependence of the denominator in (35) we cannot compare the overall strength of $M_{R}^{L F}$ and $M_{R}^{t r}$. In general, in the absence of the enhancement due to an actual triple resonance, the two contributions should have the same order of magnitude 12]. Experimentally, however, the overall intensity (and, to some extent, the form) of the $A_{1 g}$ Raman profile demonstrates a substantial dependence on the incident photon frequency. Besides, as we noted above, the Loudon-Fleury result for the $A_{1 g}$ intensity at the threshold is more than 1000 times smaller than the $B_{1 g}$ intensity at its maximum, while the experimental intensity ratio is about 40 times smaller in the vicinity of the triple resonance in $B_{1 g}$ geometry, and even far smaller away from the resonance. It is therefore very likely that the Loudon-Fleury contribution to the $A_{1 g}$ intensity is just a minor correction to the intensity given by the triple resonance diagram. Notice also that $M_{R}^{L F}$ has exactly the same form as the second term in $M_{R}^{t r}$, and its inclusion will just change the relative strength of the two terms in (36). 


\section{DISCUSSION}

We first summarize our results. We considered in this paper two-magnon Raman scattering in a two-layer Hubbard model at half-filling. We applied the SDW formalism and derived diagrammatically the Loudon-Fleury Hamiltonian for the interaction between light and spin degrees of freedom. We found that in a two-layer system, the scattering in $A_{1 g}$ geometry is finite already in the Loudon-Fleury approximation. Without final state interaction, the intensity in this channel scales as $\Omega^{3}$ at low frequencies. The magnon-magnon interaction effects are numerically small, but nevertheless they change the frequency dependence to $I_{A_{1 g}} \propto \Omega$ at small frequencies. Furthermore, there is a very strong resonance near $\Omega=4\left(J_{1} J_{2}\right)^{1 / 2}$, when a second scattering channel opens up. At resonance, the amplitude of the $A_{1 g}$ signal is larger than the amplitude of the $B_{1 g}$ signal at the same frequency. We also argued that in the resonance regime relevant to experiments on parent high- $T_{c}$ compounds, there is no enhancement of the peak intensity in $A_{1 g}$ geometry due to the actual triple resonance. Nevertheless, the diagram with three resonant denominators is dominant in this regime as it yields a finite $A_{1 g}$ intensity even without $J_{2}$.

In Fig. 8 we present the experimental data for the $A_{1 g}$ Raman intensity for the singlelayer $\mathrm{Sr}_{2} \mathrm{CuO}_{2} \mathrm{Cl}_{2}$ and the double-layer $\mathrm{YBa}_{2} \mathrm{Cu}_{3} \mathrm{O}_{6.1}$ compounds 36. We see that at transferred frequencies $\omega \geq 2000 \mathrm{~cm}^{-1}$, the intensity profiles in the two compounds are similar. The sharp peaks at the low energy tail of the two-magnon band are due to resonant multi-phonon scattering that becomes strongly enhanced for excitations close to $2 \Delta$. Despite the overall similarity of the two figures, there are clear differences at low frequencies. The intensity in a single-layer compound continues to decrease at frequencies smaller than the resonance frequencies for phonon scattering, while the intensity for a two-layer compound flattens at frequencies somewhat larger than the resonance frequencies for phonon scattering, and remains flat down to the smallest measured frequencies.

At the moment, we do not understand the origin of the background contribution to the scattering in $Y B C O$, but it is unlikely that this background contribution is related to 
scattering in a half-filled insulator. A more likely possibility is that the background is due to the fact that the measured $Y B C O$ compound has some finite amount of holes. In any event, however, we see that the intensity flattens at about $1800 \mathrm{~cm}^{-1}$, and is rather flat at even lower frequencies.

This behavior is consistent with our result for the resonant regime where the experiments have been performed: the $A_{1 g}$ intensity evaluated for $J_{2}=0.1 J_{1}$ flattens at about $1.8 J_{1} \sim 1800 \mathrm{~cm}^{-1}$, and is roughly two times flatter at low frequencies than in a single-layer compound. Indeed, the theory also predicts that there should be a jump in the intensity at the threshold frequency. However, if we associate the onset of flattening with $1800 \mathrm{~cm}^{-1}$, we find that the jump occurs at about $1200 \mathrm{~cm}^{-1}$, i.e., right at the frequencies where the Raman signal is presumably dominated by phonon scattering, so there are little chances to observe this jump directly. We therefore believe that $J_{2} \sim 0.1 J_{1}$ is a reasonable though indirect estimate of $J_{2}$.

Notice that $J_{2} \sim 0.1 J_{1} \sim 150 \mathrm{~K}$ is consistent with the estimate obtained from the analysis of the NMR data in similar systems. More rigorously, we can place the upper bound on possible values of $J_{2}$ because whatever the interpretation of the low frequency measurements is, the data above $2000 \mathrm{~cm}^{-1}$ clearly show no influence of the interlayer coupling. This in turn implies that in any event, the threshold frequency is lower than $2000 \mathrm{~cm}^{-1}$, or $J_{2}<0.25 J_{1}$. Even this estimate is substantially smaller than $J_{2} \sim 0.55 J_{1}$ extracted from the data of infrared transmission and reflection measurements in $Y B C O$ [20]. Given that inelastic neutron scattering measurements were unable to detect the optical spin wave branch in antiferromagnetic $Y B C O$ up to $60 \mathrm{meV}$, it was argued [17] that the gap, $2\left(J_{1} J_{2}\right)^{1 / 2}$, should be larger than $60 \mathrm{meV}$. We therefore conclude that $J_{2}$ must be in the energy range $8 \mathrm{meV}<$ $J_{2}<30 \mathrm{meV}$.

\section{Acknowledgments}

It is our pleasure to thank D. Frenkel, M.V. Klein, A. Millis and H. Monien for helpful conversations. A.C. is an A.P. Sloan fellow. A.P.K. gratefully acknowledges support by the Deutsche Forschungsgemeinschaft through a Heisenberg grant and the Sonderforschungs- 
bereich 341. G.B. was supported by NSF cooperative agreement DMR 91-20000 through the Science and Technology Center for Superconductivity.

\section{APPENDIX A: DERIVATION OF THE ENERGY DISPERSIONS OF THE QUASIPARTICLE CONDUCTION AND VALENCE BANDS}

In this Appendix we derive the dispersions for the valence and conduction fermions in the one-band double-layer Hubbard model at half-filling. After Fourier transformation to momentum space the Hubbard Hamiltonian Eq. (1) takes the form

$$
\begin{aligned}
H= & \sum_{\mathbf{k}, \sigma}\left(-4 t \nu_{\mathbf{k}}\right)\left(c_{\mathbf{k}, \sigma}^{\dagger} c_{\mathbf{k}, \sigma}+d_{\mathbf{k}, \sigma}^{\dagger} d_{\mathbf{k}, \sigma}\right)-t^{\prime} \sum_{\mathbf{k}}\left(c_{\mathbf{k}, \sigma}^{\dagger} d_{\mathbf{k}, \sigma}+d_{\mathbf{k}, \sigma}^{\dagger} c_{\mathbf{k}, \sigma}\right) \\
& +\frac{U}{2 N} \sum_{\mathbf{k}, \mathbf{k}^{\prime}, \mathbf{q}, \sigma}\left(c_{\mathbf{k}^{\prime}+\mathbf{q}, \sigma}^{\dagger} c_{\mathbf{k},-\sigma}^{\dagger} c_{-\mathbf{k}^{\prime},-\sigma} c_{\mathbf{k}+\mathbf{q}, \sigma}+d_{\mathbf{k}^{\prime}+\mathbf{q}, \sigma}^{\dagger} d_{\mathbf{k},-\sigma}^{\dagger} d_{-\mathbf{k}^{\prime},-\sigma} d_{\mathbf{k}+\mathbf{q}, \sigma}\right) .
\end{aligned}
$$

The presence of long range antiferromagnetic SDW order in the ground state implies that

$$
\begin{aligned}
& \frac{1}{N} \sum_{\mathbf{k}}\left\langle c_{\mathbf{k}+\pi, \uparrow}^{\dagger} c_{\mathbf{k}, \uparrow}\right\rangle=-\frac{1}{N} \sum_{\mathbf{k}}\left\langle c_{\mathbf{k}+\pi, \downarrow}^{\dagger} c_{\mathbf{k}, \downarrow}\right\rangle=m \neq 0 \\
& \frac{1}{N} \sum_{\mathbf{k}}\left\langle d_{\mathbf{k}+\pi, \downarrow}^{\dagger} d_{\mathbf{k}, \downarrow}\right\rangle=-\frac{1}{N} \sum_{\mathbf{k}}\left\langle d_{\mathbf{k}+\pi, \uparrow}^{\dagger} d_{\mathbf{k}, \uparrow}\right\rangle=m .
\end{aligned}
$$

Introducing the linear combinations

$$
\alpha_{\mathbf{k}, \sigma}=\frac{1}{\sqrt{2}}\left(c_{\mathbf{k} \sigma}+d_{\mathbf{k} \sigma}\right), \quad \beta_{\mathbf{k} \sigma}=\frac{1}{\sqrt{2}}\left(c_{\mathbf{k} \sigma}-d_{\mathbf{k} \sigma}\right)
$$

and decoupling the interaction term with the expectation values of (A2), the Hubbard Hamiltonian (A1) turns into

$$
\begin{aligned}
H=\frac{1}{N} \sum_{\mathbf{k}, \sigma}^{\prime}\{ & \left(-4 t \nu_{\mathbf{k}}-t^{\prime}\right)\left(\alpha_{\mathbf{k}, \sigma}^{\dagger} \alpha_{\mathbf{k}, \sigma}-\beta_{\mathbf{k}+\pi, \sigma}^{\dagger} \beta_{\mathbf{k}+\pi, \sigma}\right)-U m \operatorname{sgn}(\sigma)\left[\alpha_{\mathbf{k}, \sigma}^{\dagger} \beta_{\mathbf{k}+\pi, \sigma}+\beta_{\mathbf{k}+\pi, \sigma}^{\dagger} \alpha_{\mathbf{k}, \sigma}\right] \\
& \left.+\left(4 t \nu_{\mathbf{k}}-t^{\prime}\right)\left(\alpha_{\mathbf{k}+\pi, \sigma}^{\dagger} \alpha_{\mathbf{k}+\pi, \sigma}-\beta_{\mathbf{k}, \sigma}^{\dagger} \beta_{\mathbf{k}, \sigma}\right)-U m \operatorname{sgn}(\sigma)\left[\alpha_{\mathbf{k}+\pi, \sigma}^{\dagger} \beta_{\mathbf{k}, \sigma}+\beta_{\mathbf{k}, \sigma}^{\dagger} \alpha_{\mathbf{k}+\pi, \sigma}\right]\right\},
\end{aligned}
$$

where the primed momentum sum is restricted to the magnetic Brillouin zone. Two

separate Bogolyubov transformations applied to the first and the second part of the Hamiltonian (A4) yield two pairs of conduction and valence bands with the dispersions 
$\pm E_{\mathbf{k}}^{+}= \pm \sqrt{\left(4 t \nu_{\mathbf{k}}+t^{\prime}\right)^{2}+\Delta^{2}}$ and $\pm E_{\mathbf{k}}^{-}= \pm \sqrt{\left(4 t \nu_{\mathbf{k}}-t^{\prime}\right)^{2}+\Delta^{2}}$. The self-consistency condition for $\Delta=U m$ requires that

$$
\frac{1}{U}=\frac{1}{2 N} \sum_{\mathbf{k}}^{\prime}\left[\frac{1}{E_{\mathbf{k}}^{+}}+\frac{1}{E_{\mathbf{k}}^{-}}\right]
$$

\section{APPENDIX B: TRANSVERSE SUSCEPTIBILITIES IN A DOUBLE-LAYER ANTIFERROMAGNET}

The dynamic, transverse spin susceptibility is obtained from the time ordered correlation function

$$
\chi_{\alpha \beta}^{+-}\left(\mathbf{q}, \mathbf{q}^{\prime}, t\right)=i<T S_{\mathbf{q}, \alpha}^{+}(t) S_{-\mathbf{q}^{\prime}, \beta}^{-}(0)>
$$

where the indices $\alpha, \beta=1,2$ denote the layer. In terms of fermion operators the spin raising and lowering operators $S_{\mathbf{q}, \alpha}^{ \pm}$are expressed through

$$
\mathbf{S}_{\mathbf{q}, 1}=\frac{1}{N} \sum_{\mathbf{k}} \sum_{\mu, \nu} c_{\mathbf{k}+\mathbf{q}, \mu}^{\dagger} \vec{\sigma}_{\mu, \nu} c_{\mathbf{k}, \nu}, \quad \mathbf{S}_{\mathbf{q}, 2}=\frac{1}{N} \sum_{\mathbf{k}} \sum_{\mu, \nu} d_{\mathbf{k}+\mathbf{q}, \mu}^{\dagger} \vec{\sigma}_{\mu, \nu} d_{\mathbf{k}, \nu}
$$

where $\vec{\sigma}$ are the Pauli matrices and the $c$ and $d$ operators describe the electrons from layer 1 and 2, respectively. Summing the RPA ladder diagram series for the transverse susceptibilities as described in detail in the literature for a single-layer system [24, 34, 35] leads to the following results (for $S=1 / 2$ )

$$
\begin{aligned}
\chi_{11}^{+-}(\mathbf{q}, \mathbf{q}, \omega) & =-\frac{J_{1}\left(1-\nu_{\mathbf{q}}\right)}{\omega^{2}-\Omega_{1}^{2}(\mathbf{q})+i \delta}-\frac{J_{1}\left(1-\nu_{\mathbf{q}}+J_{2} / 2 J_{1}\right)}{\omega^{2}-\Omega_{2}^{2}(\mathbf{q})+i \delta} \\
\chi_{11}^{+-}(\mathbf{q}, \mathbf{q}+\mathbf{Q}, \omega) & =\frac{1}{2}\left[\frac{\omega}{\omega^{2}-\Omega_{1}^{2}(\mathbf{q})+i \delta}+\frac{\omega}{\omega^{2}-\Omega_{2}^{2}(\mathbf{q})+i \delta}\right],
\end{aligned}
$$

when the spins are from the same layer $\left(\chi_{11}^{+-}=\chi_{22}^{+-}\right)$, and

$$
\begin{aligned}
\chi_{12}^{+-}(\mathbf{q}, \mathbf{q}, \omega) & =-\frac{J_{1}\left(1-\nu_{\mathbf{q}}\right)}{\omega^{2}-\Omega_{1}^{2}(\mathbf{q})+i \delta}+\frac{J_{1}\left(1-\nu_{\mathbf{q}}+J_{2} / 2 J_{1}\right)}{\omega^{2}-\Omega_{2}^{2}(\mathbf{q})+i \delta} \\
\chi_{12}^{+-}(\mathbf{q}, \mathbf{q}+\mathbf{Q}, \omega) & =\frac{1}{2}\left[\frac{\omega}{\omega^{2}-\Omega_{1}^{2}(\mathbf{q})+i \delta}-\frac{\omega}{\omega^{2}-\Omega_{2}^{2}(\mathbf{q})+i \delta}\right],
\end{aligned}
$$


when the spins are from different layers $\left(\chi_{12}^{+-}=\chi_{21}^{+-}\right)$. The poles of the susceptibilities are at $\Omega_{1}(\mathbf{q})$ and $\Omega_{2}(\mathbf{q}) \equiv \Omega_{1}(\mathbf{q}+\mathbf{Q})$, as they should. Using these results, one can construct the effective Hamiltonian for the magnon-fermion interaction Eq. (7) by the requirement that it reproduces the forms of all these susceptibilities.

\section{APPENDIX C: RAMAN INTENSITIES WITHOUT MAGNON-MAGNON INTERACTION}

In this Appendix we present the closed forms of $I_{A_{1 g}}$ and $I_{B_{1 g}}$ without final state magnonmagnon interaction. The actual calculations have been performed to leading order in $1 / S$. Here, we present the results for $S=1 / 2$. We introduce the short notation $\tilde{\Omega}=\Omega / 2 J_{1}$ and

$$
\begin{aligned}
a & =\frac{\left(16 t^{2}\right)^{2}}{J_{1} \pi^{2} \hbar}\left[\frac{2 \Delta}{4 \Delta^{2}-\Omega^{2}} \frac{J_{2}}{4 J_{1}}\right]^{2}, \quad b=\frac{\left(16 t^{2}\right)^{2}}{J_{1} \pi^{2} \hbar}\left[\frac{2 \Delta}{4 \Delta^{2}-\Omega^{2}}\right]^{2}\left(1+\frac{J_{2}}{4 J_{1}}\right), \\
t_{+} & =\frac{1-\nu_{+}}{1+\nu_{+}}, \quad t_{-}=\frac{1+\nu_{-}}{1-\nu_{-}}, \quad g(\tilde{\Omega})=\frac{1}{2 \tilde{\Omega}} \frac{1}{\sqrt{\left(1+J_{2} / 4 J_{1}\right)^{2}-\tilde{\Omega}^{2} / 4}},
\end{aligned}
$$

where

$$
\nu_{+,-}=-\left(J_{2} / 4 J_{1}\right) \pm \sqrt{\left.\left(1+J_{2} / 4 J_{1}\right)\right)^{2}-\tilde{\Omega}^{2} / 4} .
$$

For $A_{1 g}$ scattering geometry we then obtain:

i) for $0 \leq \tilde{\Omega} \leq 2 \sqrt{J_{2} / J_{1}}$

$$
I_{A_{1 g}}(\Omega)=a g(\tilde{\Omega}) \frac{\left(1-\nu_{+}\right)^{2}}{1+\nu_{+}} K\left(t_{+}\right)
$$

where $K$ is the complete elliptic integral of the first kind;

ii) for $2 \sqrt{J_{2} / J_{1}} \leq \tilde{\Omega} \leq 2 \sqrt{1+J_{2} / 2 J_{1}}$

$$
I_{A_{1 g}}(\Omega)=\operatorname{ag}(\tilde{\Omega})\left\{\frac{\left(1-\nu_{+}\right)^{2}}{1+\nu_{+}} K\left(t_{+}\right)+\left(1-\nu_{-}\right) K\left(t_{-}\right)\right\} ;
$$

iii) for $2 \sqrt{1+J_{2} / 2 J_{1}} \leq \tilde{\Omega} \leq 2\left(1+J_{2} / 4 J_{1}\right)$

$$
I_{A_{1 g}}(\Omega)=a g(\tilde{\Omega})\left\{\left(1-\nu_{+}\right) K\left(t_{+}\right)+\left(1-\nu_{-}\right) K\left(t_{-}\right)\right\} .
$$

For $B_{1 g}$ scattering geometry we obtain

i) for $0 \leq \tilde{\Omega} \leq 2 \sqrt{J_{2} / J_{1}}$ 


$$
I_{B_{1 g}}(\Omega)=b g(\tilde{\Omega})\left(1+\nu_{+}\right)\left[K\left(t_{+}\right)-E\left(t_{+}\right)\right]
$$

where $E$ is the complete elliptic integral of the second kind;

ii) for $2 \sqrt{J_{2} / J_{1}} \leq \tilde{\Omega} \leq 2 \sqrt{1+J_{2} / 2 J_{1}}$

$$
I_{B_{1 g}}(\Omega)=b g(\tilde{\Omega})\left\{\left(1+\nu_{+}\right)\left[K\left(t_{+}\right)-E\left(t_{+}\right)\right]+\left(1-\nu_{-}\right)\left[K\left(t_{-}\right)-E\left(t_{-}\right)\right]\right\}
$$

iii) for $2 \sqrt{1+J_{2} / 2 J_{1}} \leq \tilde{\Omega} \leq 2\left(1+J_{2} / 4 J_{1}\right)$

$$
I_{B_{1 g}}(\Omega)=b g(\tilde{\Omega})\left\{\left(1-\nu_{+}\right)\left[K\left(t_{+}\right)-E\left(t_{+}\right)\right]+\left(1-\nu_{-}\right)\left[K\left(t_{-}\right)-E\left(t_{-}\right)\right]\right\} .
$$

\section{APPENDIX D: RAMAN INTENSITY WITH MAGNON-MAGNON INTERACTION}

In this Appendix we outline our calculations of the full Raman intensity with final state interaction. Considering repeated two-magnon scattering we sum the corresponding series of ladder diagrams (see, e.g. [11]). The resulting integral equation for the full vertex function reduces to a set of algebraic equations which allows for an explicit solution. We skip the details and list here only the results.

For the Raman intensity in $A_{1 g}$ geometry we obtain

$$
I_{A_{1 g}}(\Omega) \propto \operatorname{Im} \frac{\alpha(\Omega)}{1+\frac{\gamma}{2} \alpha(\Omega)}
$$

where

$$
\gamma=J_{2}\left(4 J_{1}\right)^{2}\left(1+\frac{J_{2}}{4 J_{1}}\right), \quad \alpha(\Omega)=P^{(2)}(\Omega)+2 J_{1}\left[R^{(1)}(\Omega) E(\Omega)+\frac{J_{2}}{4 J_{1}} P^{(1)}(\Omega) A(\Omega)\right] .
$$

Here we defined

$$
\begin{aligned}
& A(\Omega)=\frac{-P^{(1)}(\Omega)+R^{(1)}(\Omega) H(\Omega)}{1+\frac{1}{2} J_{2}\left[P^{(0)}(\Omega)-R^{(0)}(\Omega) H(\Omega)\right]} \\
& H(\Omega)=\frac{2 J_{1} R^{(0)}(\Omega)}{1+2 J_{1} Q(\Omega)} \\
& E(\Omega)=-\frac{\frac{1}{2} J_{2} R^{(0)}(\Omega) A(\Omega)+R^{(1)}(\Omega)}{1+2 J_{1} Q(\Omega)}
\end{aligned}
$$


and also

$$
\begin{aligned}
P^{(m)}(\Omega) & =\frac{i}{N} \sum_{\mathbf{q}} \int \frac{d \omega}{2 \pi}\left(\frac{1-\nu_{\mathbf{q}}}{\Omega_{1}(\mathbf{q})}\right)^{m} G_{0}(\mathbf{q}, \Omega+\omega) G_{0}(-\mathbf{q},-\omega) \\
& =\frac{1}{N} \sum_{\mathbf{q}}\left(\frac{1-\nu_{\mathbf{q}}}{\Omega_{1}(\mathbf{q})}\right)^{m} \frac{1}{\Omega-2 \Omega_{1}(\mathbf{q})+i \delta} \\
R^{(m)}(\Omega) & =\frac{i}{N} \sum_{\mathbf{q}} \int \frac{d \omega}{2 \pi}\left(\frac{1-\nu_{\mathbf{q}}}{\Omega_{1}(\mathbf{q})}\right)^{m} \nu_{\mathbf{q}} G_{0}(\mathbf{q}, \Omega+\omega) G_{0}(-\mathbf{q},-\omega) \\
& =\frac{1}{N} \sum_{\mathbf{q}}\left(\frac{1-\nu_{\mathbf{q}}}{\Omega_{1}(\mathbf{q})}\right)^{m} \frac{\nu_{\mathbf{q}}}{\Omega-2 \Omega_{1}(\mathbf{q})+i \delta} \\
Q(\Omega) & =\frac{i}{N} \sum_{\mathbf{q}} \int \frac{d \omega}{2 \pi} \nu_{\mathbf{q}}^{2} G_{0}(\mathbf{q}, \Omega+\omega) G_{0}(-\mathbf{q},-\omega) \\
& =\frac{1}{N} \sum_{\mathbf{q}} \frac{\nu_{\mathbf{q}}^{2}}{\Omega-2 \Omega_{1}(\mathbf{q})+i \delta} .
\end{aligned}
$$

where $G_{0}(\mathbf{q}, \omega)=\left(\omega-\Omega_{1}(\mathbf{q})+i \delta\right)^{-1}$ is the non-interacting spin wave propagator.

For $B_{1 g}$ geometry we find

$$
I_{B_{1 g}}(\Omega) \propto \operatorname{Im} \frac{L^{(2)}(\Omega)-4 J_{1}\left[L^{(1)}(\Omega) L^{(1)}(\Omega)-L^{(0)}(\Omega) L^{(2)}(\Omega)\right]}{1+4 J_{1} L^{(0)}(\Omega)+16 J_{1}^{2}\left(J_{2}+4 J_{1}\right)^{2}\left[L^{(0)}(\Omega) L^{(2)}(\Omega)-L^{(1)}(\Omega) L^{(1)}(\Omega)+\frac{L^{(2)}(\Omega)}{4 J_{1}}\right]}
$$

where

$$
\begin{aligned}
L^{(m)}(\Omega) & =\frac{i}{N} \sum_{\mathbf{q}} \int \frac{d \omega}{2 \pi} \frac{\tilde{\nu}_{\mathbf{q}}^{2}}{\Omega_{1}^{m}(\mathbf{q})} G_{0}(\mathbf{q}, \Omega+\omega) G_{0}(-\mathbf{q},-\omega) \\
& =\frac{1}{N} \sum_{\mathbf{q}} \frac{\tilde{\nu}_{\mathbf{q}}^{2}}{\Omega_{1}^{m}(\mathbf{q})} \frac{1}{\Omega-2 \Omega_{1}(\mathbf{q})+i \delta} .
\end{aligned}
$$

This form is similar to the result for a single layer. The plots of the full intensities are presented in Figs. 专 and 5 . 


\section{REFERENCES}

[1] J.G. Bednorz and K.A. Müller, Z. Phys. B 64, 189 (1986); Rev. Mod. Phys. 60, 585 (1988).

[2] P.W. Anderson, in Superconductivity, Proceedings of the ICTP Spring College 1992, P. Butcher and Y. Lu, Eds. (World Scientific Press, Singapore, 1993); S. Chakravarty, A. Sudbø, P.W. Anderson, and S. Strong, Science 261, 337 (1993); A. Sudbø, S. Chakravarty, S. Strong, and P.W. Anderson, Phys. Rev. B 49, 12245 (1994).

[3] B.L. Altshuler, and L.B. Ioffe, Solid State Comm. 82, 253 (1992); B.L. Altshuler, L.B. Ioffe, A.I. Larkin, and A.J. Millis, JETP Lett. 59, 65 (1994) and unpublished.

[4] A.J. Millis and H. Monien, Phys. Rev. Lett. 70, 2810 (1993); Phys. Rev. Lett. 71, 210(E) (1993); Phys. Rev. B 50, 16606 (1994).

[5] M. Ubbens and P. Lee, Phys. Rev. B 50, 438 (1994).

[6] M. Takigawa, A.P. Reyes, P.C. Hammel, J.D. Thompson, R.H. Heffner, Z. Fisk, and K.C. Ott, Phys. Rev. B 43, 247 (1991).

[7] R. Merlin, Journal de Physique (Paris) Colloque 41, C5-233 (1980).

[8] M.G. Cottam and D.L. Lockwood, Light Scattering in Magnetic Solids (Wiley, New York, 1986).

[9] R.R.P. Singh, Comments Cond. Mat. Phys. 15, 99 (1990).

[10] R.R.P. Singh, P.A. Fleury, K.B. Lyons, and P.E. Sulewski, Phys. Rev. Lett. 62, 2736 (1989).

[11] C.M. Canali and S.M. Girvin, Phys. Rev. B. 45, 7127 (1992).

[12] A.V. Chubukov and D.M. Frenkel, Phys. Rev. Lett. 74, 3057 (1995); Phys. Rev. B 52, $9760(1995)$. 
[13] Strictly speaking, this scattering geometry corresponds to $B_{1 g}+A_{2 g}$, however, we obtained in our theoretical analysis that the $A_{2 g}$ scattering is zero. Experimentally it was found that the signal in $A_{2 g}$ channel is very weak.

[14] K.B. Lyons, P.A. Fleury, L.T. Schneemeyer, and J.V. Waszczak, Phys. Rev. Lett. 60, $732(1988)$.

[15] This scattering geometry actually yields scattering in the $A_{1 g}$ and $B_{2 g}$ channels. However, the $B_{2 g}$ intensity is relatively weak and we will assume that it can be neglected compared to the $A_{1 g}$ intensity.

[16] P.A. Fleury and R. Loudon, Phys. Rev. 166, 514 (1968).

[17] S. Shamoto, M. Sato, J.M. Tranquada, B.J. Sternlieb, and G. Shirane, Phys. Rev. B 48, 13817 (1993); S.M. Hayden et al, Phys. Rev. Lett., 66, 821 (1991); ibid 67, 3622 (1991).

[18] A.J. Millis and H. Monien, preprint.

[19] R. Stern, M. Mali, I. Mangelschots, J. Roos, D. Brinkmann, J.-Y. Genoud, T. Graf, and J. Muller, Phys. Rev. B 50, 426 (1994); R. Stern, M. Mali, J. Roos, and D. Brinkmann, Phys. Rev. B 51, 15478 (1995).

[20] M. Grüninger, J. Münzel, A. Gaymann, A. Zibold, H.P. Geserich, and T. Kopp, preprint.

[21] F. Barriquand and G.A. Sawatzky, Phys. Rev. B 50, 16649 (1994).

[22] See also L. Marville, Ph.D. thesis, MIT, 1992, unpublished.

[23] B.S. Shastry and B.I. Shraiman, Phys. Rev. Lett. 65, 1068 (1990); Int. J. Mod. Phys. B 5, 365 (1991).

[24] J.R. Schrieffer, X.G. Wen, and S.C. Zhang, Phys. Rev. B 41, 11663 (1989).

[25] We do not consider a case when the light is polarized along the direction perpendicular 
to the planes.

[26] I. Affleck and F.D.M. Haldane, Phys. Rev. B 36, 5291, (1987).

[27] A.V. Chubukov and K. Musaelian, Phys. Rev. B 50, 6238 (1994).

[28] T. Matsuda and K. Hida, J. Phys. Soc. Jpn. 59, 2223 (1990).

[29] W. Hayes and R. Loudon, Scattering of Light by Crystals (Wiley-Interscience, New York, 1978) and references therein.

[30] T. Oguchi, Phys. Rev. 117, 117 (1960). For a two-layer system, the 1/S corrections were first calculated by K. Hida, J. Phys. Soc. Jpn. 61, 1013 (1992).

[31] A.V. Chubukov and D.K. Morr, Phys. Rev. B 52, 3521 (1995).

[32] R.W. Davis, S.R. Chinn, and H.J. Zeiger, Phys. Rev. B 4, 992 (1971).

[33] J. Adler, Phys. Rev. 137, 1022 (1965).

[34] A.V. Chubukov and D.M. Frenkel, Phys. Rev. B 46, 11884 (1992).

[35] W. Brenig and A.P. Kampf, Europhys. Lett. 24, 679 (1993).

[36] G. Blumberg. P. Abbamonte, M.V. Klein, L.L. Miller, W.C. Lee, and D.M. Ginsberg, preprint. 


\section{FIGURES}

FIG. 1. The system under consideration is a two layer antiferromagnet with intralayer exchange coupling $J_{1}=4 t^{2} / U$ and interlayer exchange coupling $J_{2}=4 t^{2} / U$.

FIG. 2. The diagrams which contribute to lowest order in $t / U$ to the Raman matrix element $M_{R}$. The fermions from the valence (conduction) bands are denoted by a dashed (solid) line. The emitted magnons are represented by a solid wavy line whereas the incoming $\left(\omega_{i}\right)$ and outgoing $\left(\omega_{f}\right)$ photons are given by dashed wavy lines.

FIG. 3. The Raman intensities in $A_{1 g}$ and $B_{1 g}$ geometry obtained in the Loudon-Fleury theory neglecting final state magnon-magnon interactions. The jump in the intensity in the $A_{1 g}$ geometry occurs at the frequency $2 \Omega_{1}(\mathbf{Q})=4\left(J_{2} J_{1}\right)^{1 / 2}$. The overall shape of the intensities is shifted towards higher frequencies with increasing $J_{2} / J_{1}$.

FIG. 4. The Loudon-Fleury Raman intensity in $B_{1 g}$ geometry with final state interaction for two different values of $J_{2} / J_{1}$.

FIG. 5. The Loudon-Fleury Raman intensity in $A_{1 g}$ geometry with final state interaction. The figures (a), for $J_{2} / J_{1}=0.2$, and (b), for $J_{2} / J_{1}=0.4$, include effects of magnon damping which were modeled by adding a finite imaginary part $i \delta$ to the energy denominators of the spin wave propagators. The transferred frequency in these two figures is given in units of the maximum spin wave frequency $\Omega_{\max }$. The low frequency peak in the intensity is located at a frequency somewhat smaller than $2 \Omega_{1}(\mathbf{Q})$. With increasing damping the peak frequency is gradually shifted closer to $2 \Omega_{1}(\mathbf{Q})$. For comparison, in Fig. (c) we plotted the intensity for $J_{2} / J_{1}=0.4$ without any magnon damping.

FIG. 6. The "triple-resonance" diagram which gives the dominant contribution to the Raman intensity in the resonant regime. The notations are the same as in Fig.2 
FIG. 7. The "triple-resonance" diagram contribution to the Raman intensity in $A_{1 g}$ geometry. The final state interaction is included, and $J_{2} / J_{1}=0.1$. Observe the flattening of the intensity above the threshold. There should be (in the absence of damping) a real jump at the threshold frequency - its smearing in the figure is due to limited numerical accuracy. The dashed line represents the intensity in the absence of $J_{2}$.

FIG. 8. Experimental Raman scattering data in $x^{\prime} x^{\prime}$ scattering geometry for (a) single-layer $\mathrm{Sr}_{2} \mathrm{CuO}_{2} \mathrm{Cl}_{2}$ and (b) double-layer $\mathrm{YBa}_{2} \mathrm{Cu}_{3} \mathrm{O}_{6.1}$ single crystals. The data are taken from Ref. [36. Observe the flattening of the intensity in $Y B C O$ at around $1800 \mathrm{~cm}^{-1}$. For both crystals, the excitation energy $\omega_{i}$ is $2 \Delta+2.9 J_{1}$ (the actual values are 2.33 and $2.09 \mathrm{eV}$ correspondingly). The continuum intensity at high frequencies is presumably due to multi-magnon Raman scattering, the sharp peaks at low energies are due to resonant multi-phonon scattering that becomes strongly enhanced for excitations close to $2 \Delta$. The dashed line is a fit to linear + cubic frequency dependence $I \propto\left[c\left(\omega / J_{1}\right)+\left(\omega / J_{1}\right)^{3}\right]$, where $c=1.6$ for $\mathrm{Sr}_{2} \mathrm{CuO}_{2} \mathrm{Cl}_{2}$ and 1.3 for $\mathrm{YBa}_{2} \mathrm{Cu}_{3} \mathrm{O}_{6.1}$. 

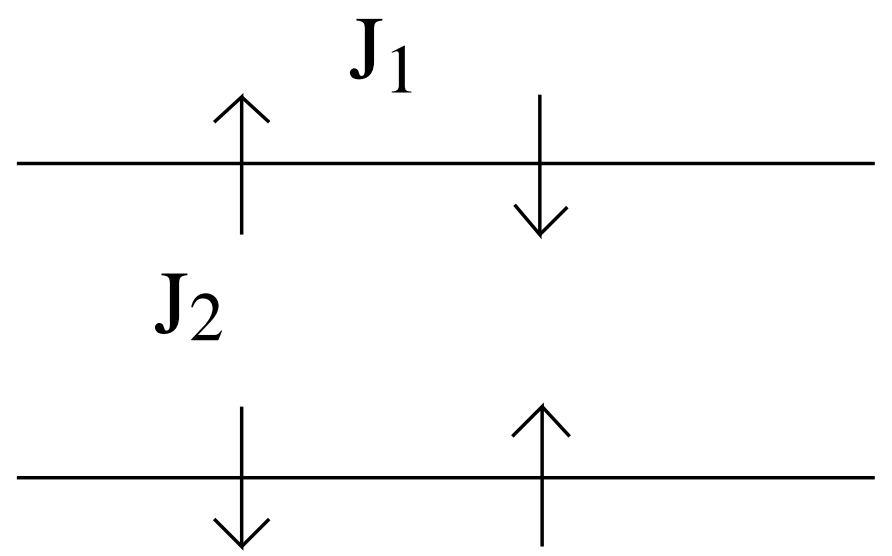

Fig.1 
(a)

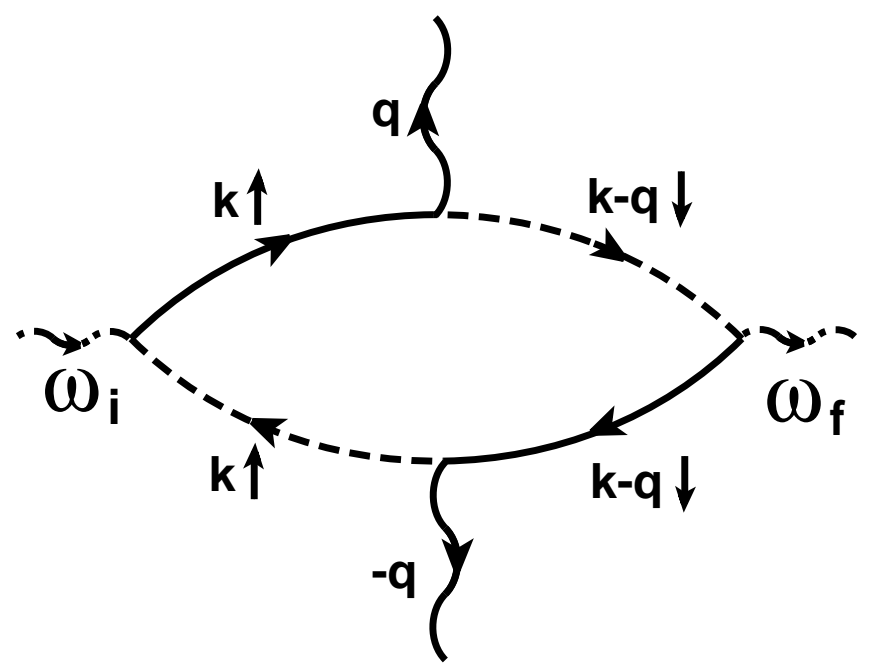

(b)

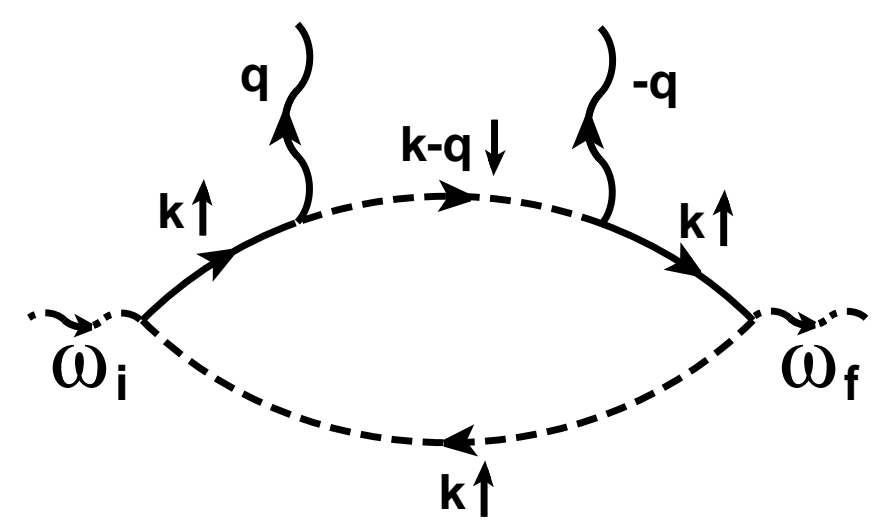

(c)

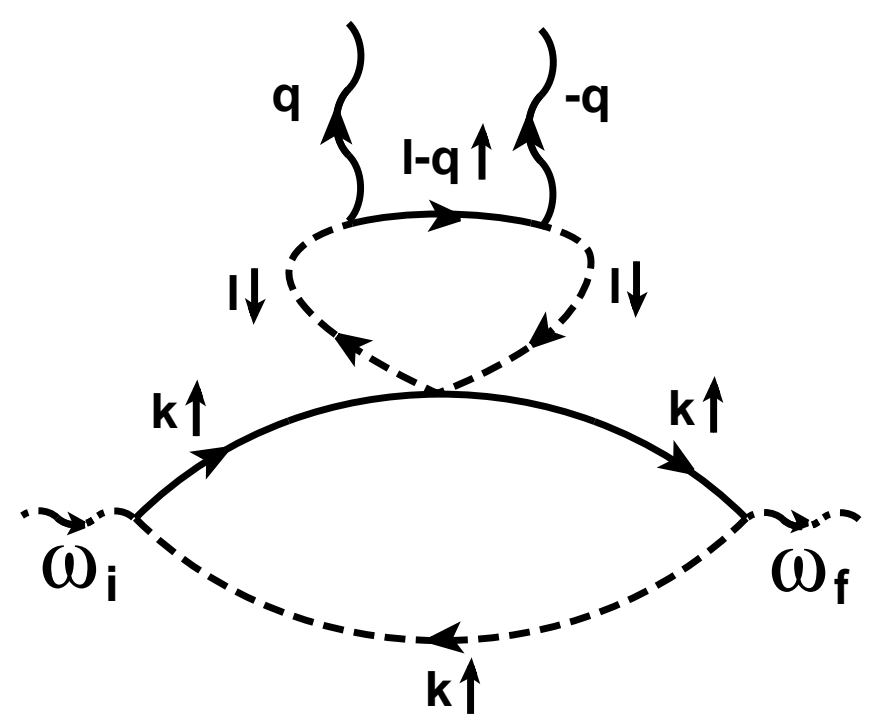

Fig.2 

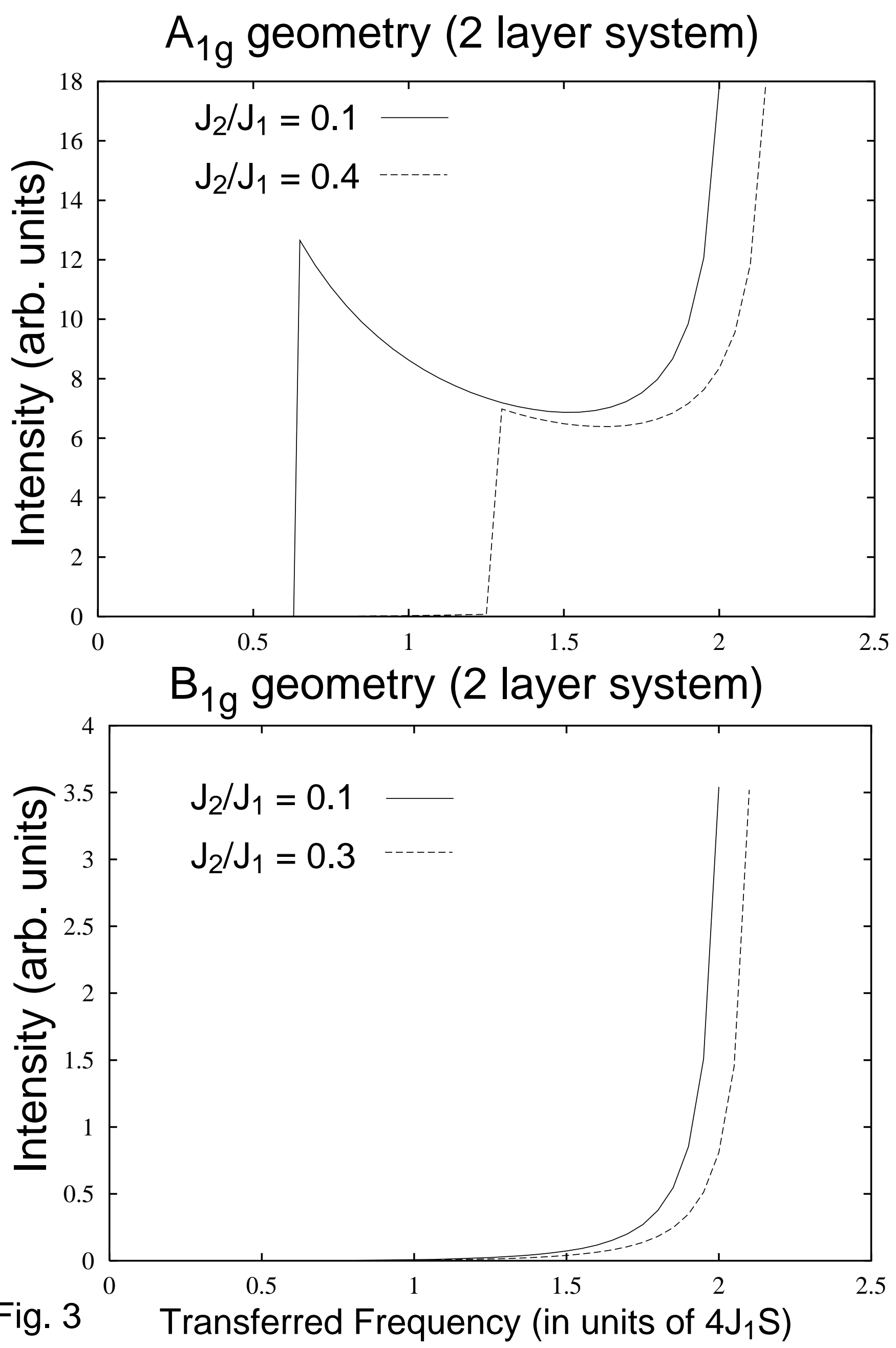
$\mathrm{B}_{1 \mathrm{~g}}$ geometry (2 layer system)

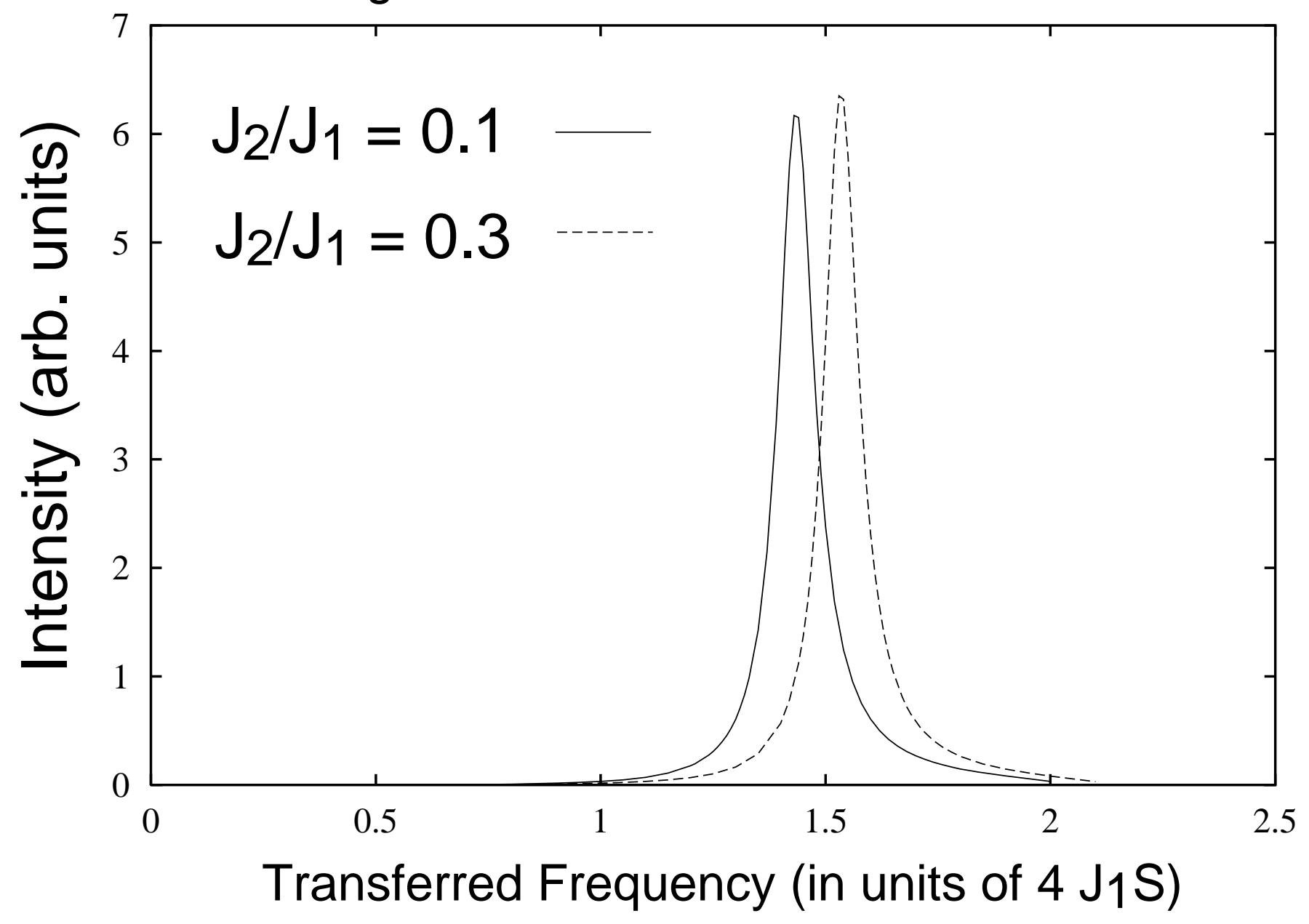

Fig. 4 


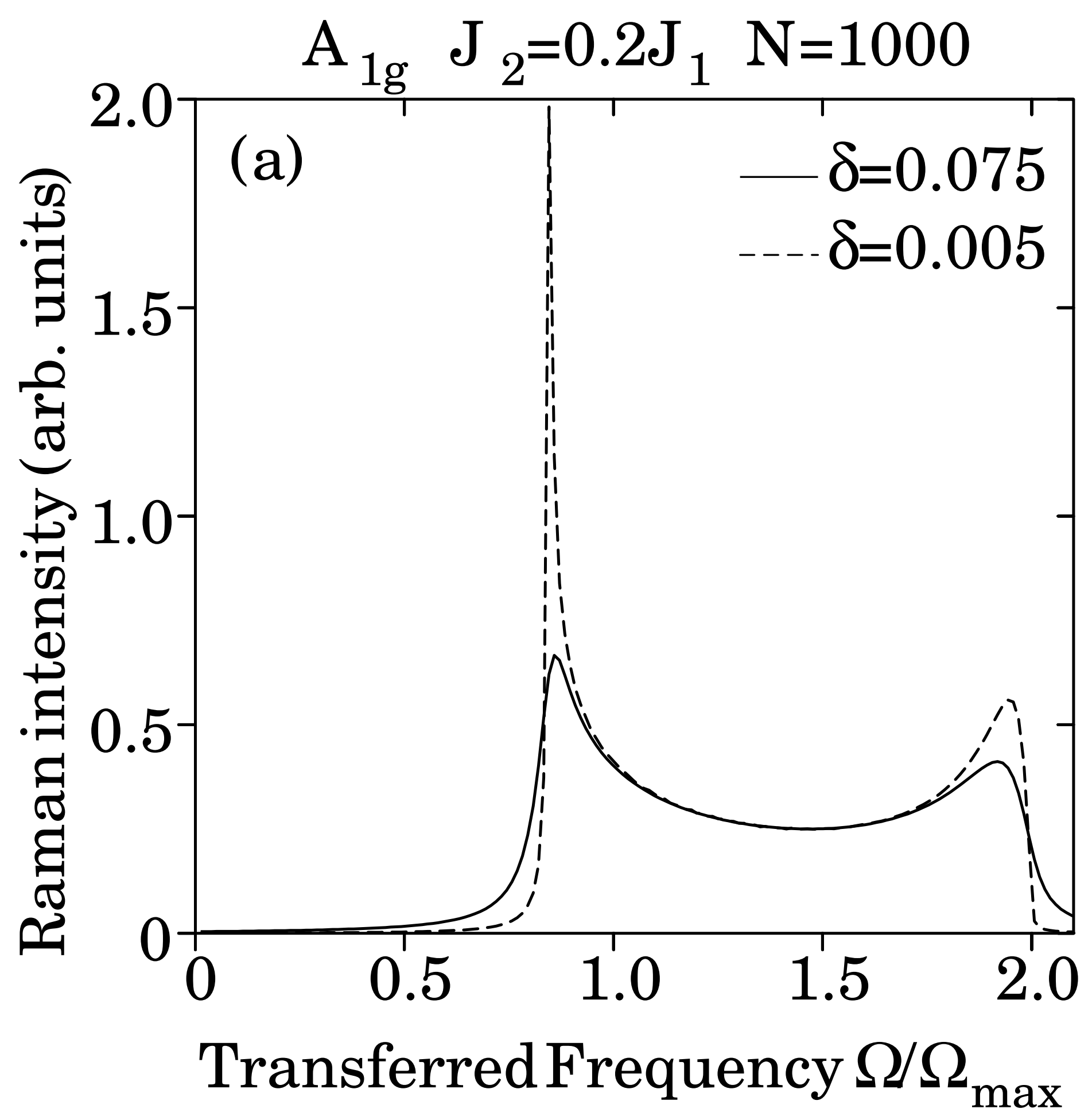




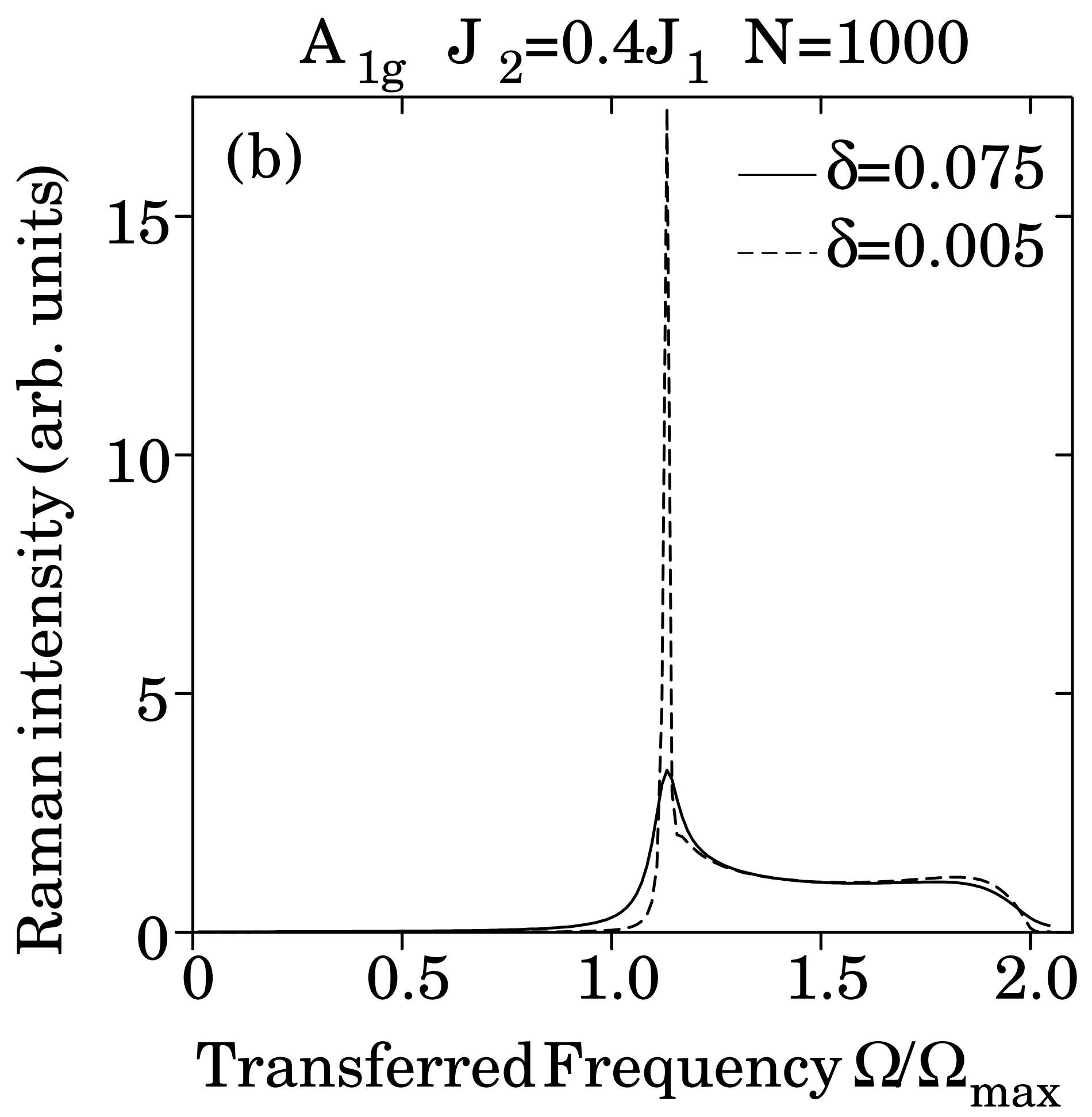


$A_{1 g}$ geometry

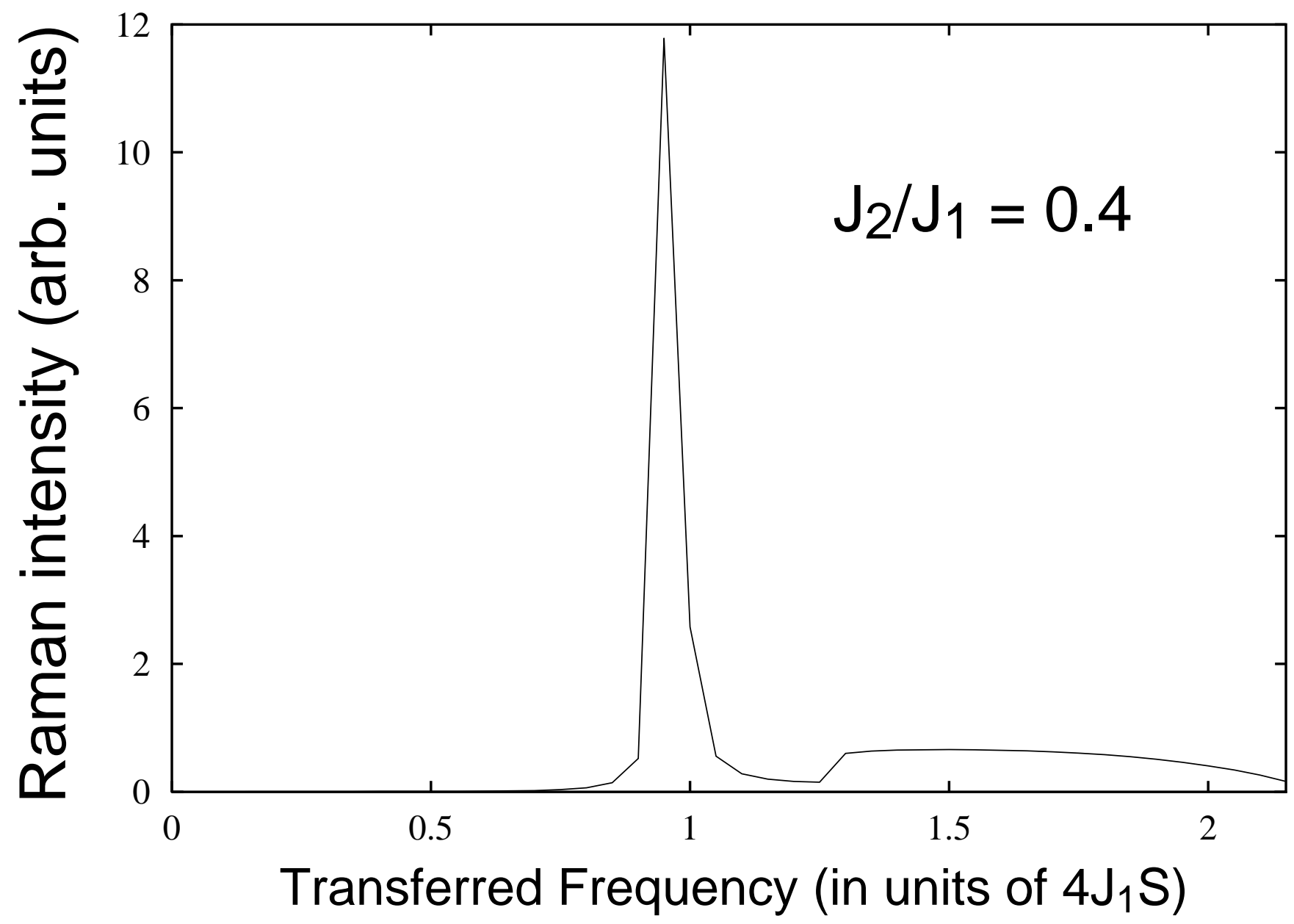

Fig. 5c 


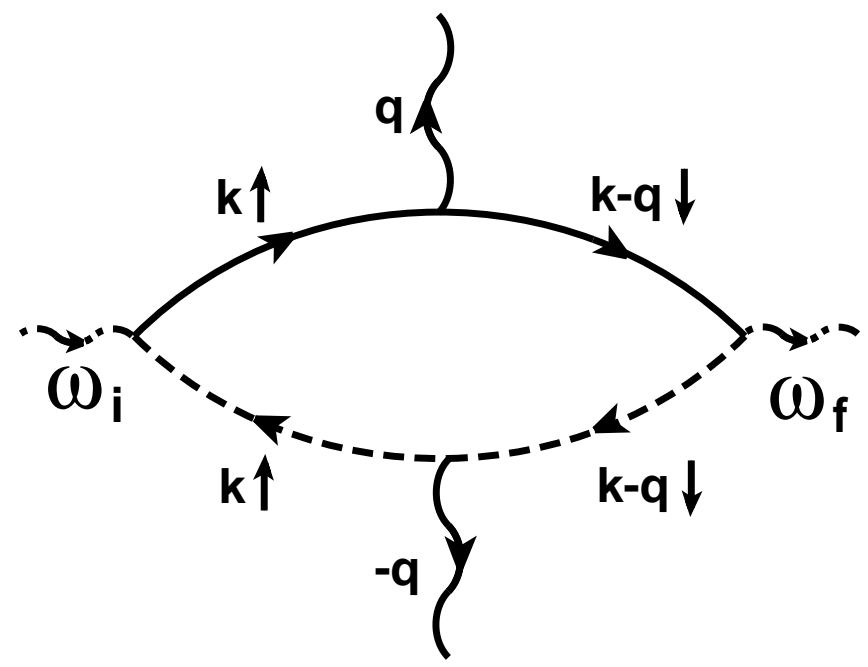

Fig.6 


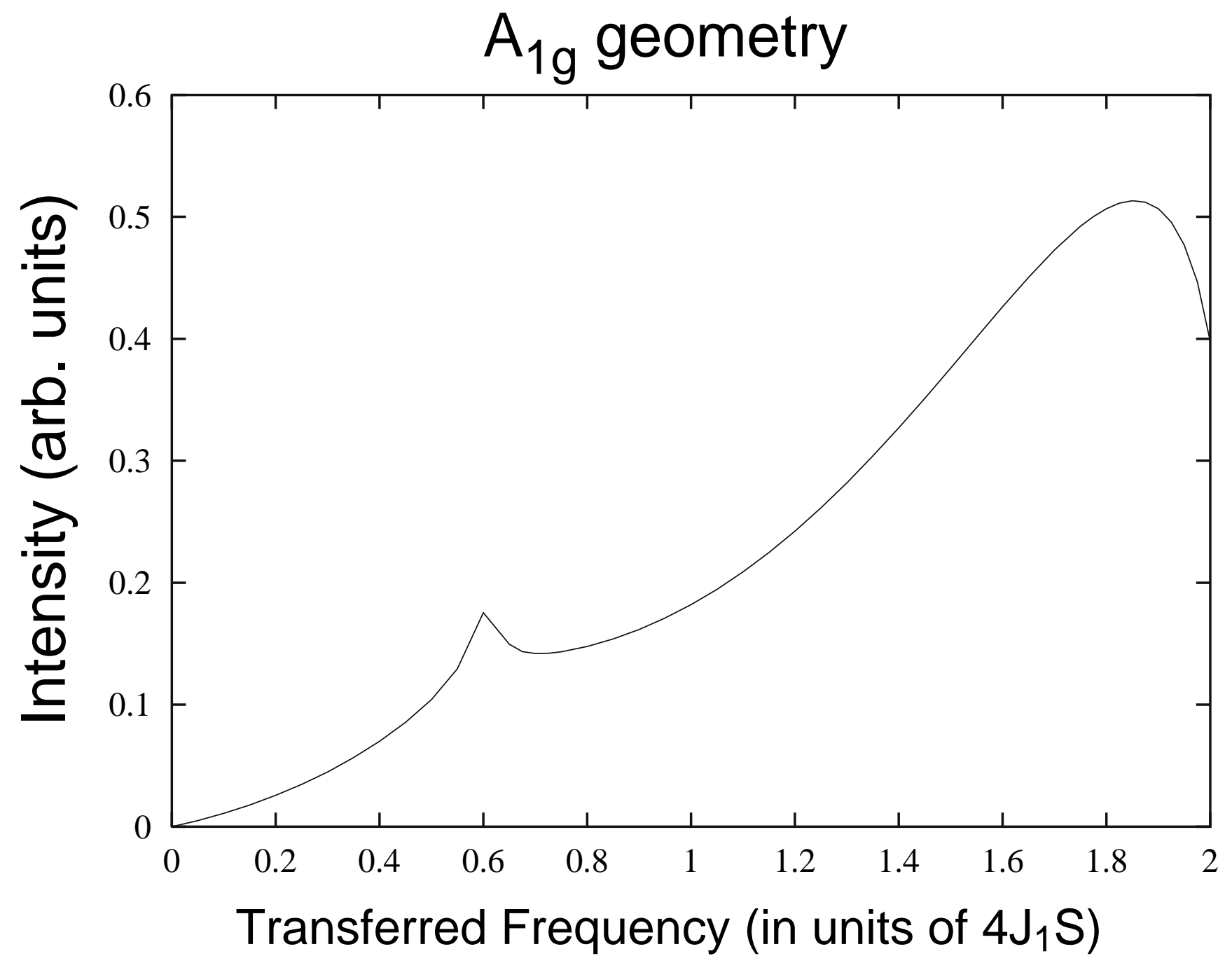

Fig. 7 


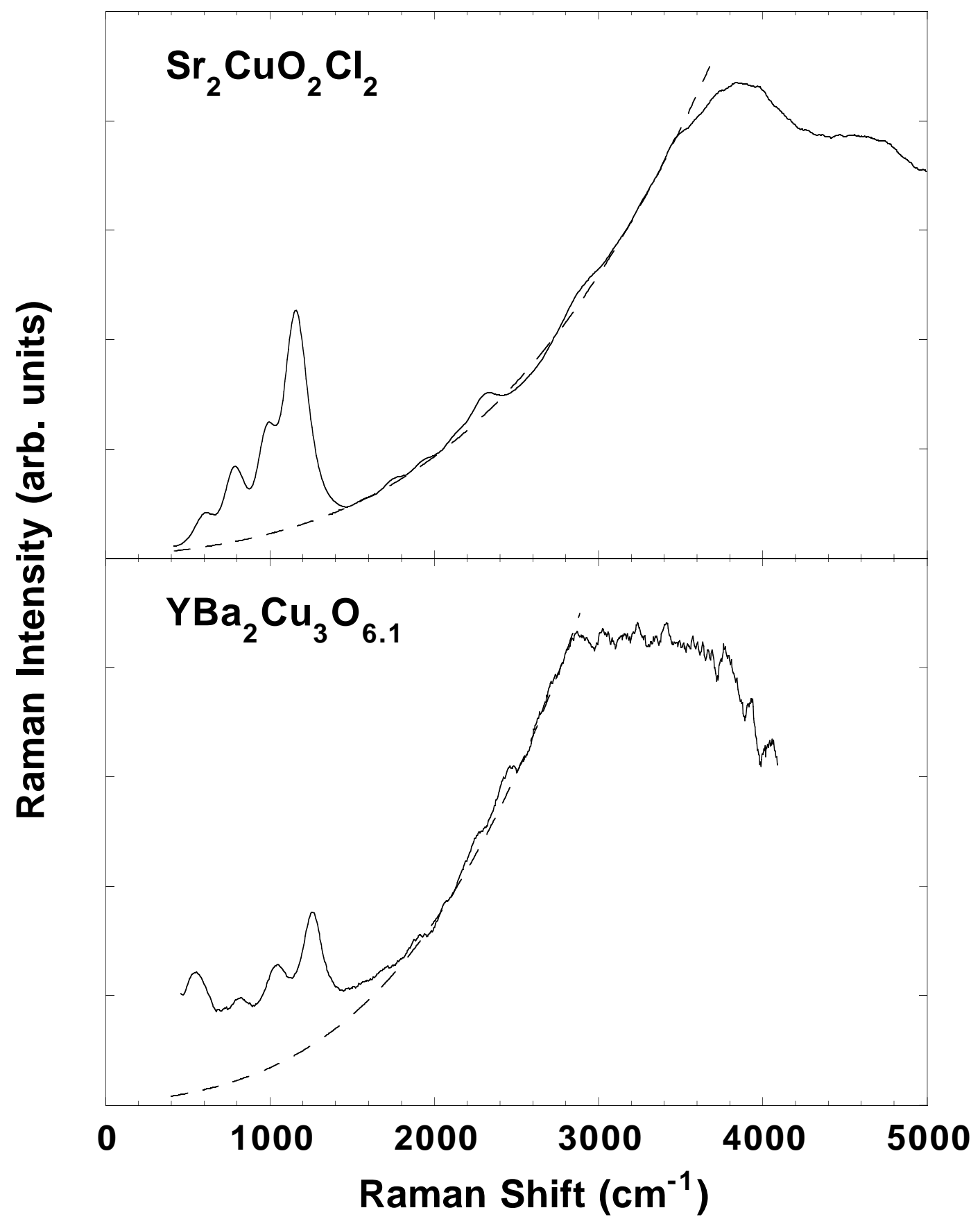

Fig. 8 